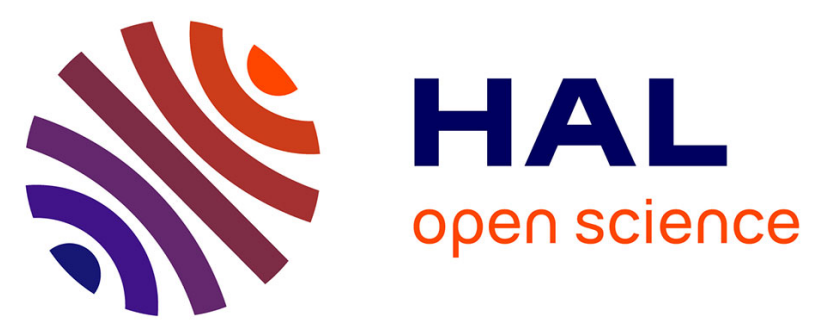

\title{
À l'occasion du centenaire des premières recherches archéologiques à Saint-Bertrand-de-Comminges (Lugdunum Convenarum), retour sur l'établissement chrétien du quartier du Plan
}

\author{
Jean Guyon, Jean-Louis Paillet
}

\section{To cite this version:}

Jean Guyon, Jean-Louis Paillet. À l'occasion du centenaire des premières recherches archéologiques à Saint-Bertrand-de-Comminges (Lugdunum Convenarum), retour sur l'établissement chrétien du quartier du Plan. Comptes-rendus des séances de l'Académie des inscriptions et belles-lettres, 2010, pp. 1265-1304. 10.3406/crai.2010.93011 . hal-01472495

\section{HAL Id: hal-01472495 \\ https://hal.science/hal-01472495}

Submitted on 20 Feb 2017

HAL is a multi-disciplinary open access archive for the deposit and dissemination of scientific research documents, whether they are published or not. The documents may come from teaching and research institutions in France or abroad, or from public or private research centers.
L'archive ouverte pluridisciplinaire HAL, est destinée au dépôt et à la diffusion de documents scientifiques de niveau recherche, publiés ou non, émanant des établissements d'enseignement et de recherche français ou étrangers, des laboratoires publics ou privés. 


\section{À l'occasion du centenaire des premières recherches} archéologiques à Saint-Bertrand-de-Comminges (Lugdunum Convenarum), retour sur l'établissement chrétien du quartier du Plan

Jean Guyon, Monsieur Jean-Louis Paillet

\section{Citer ce document / Cite this document :}

Guyon Jean, Paillet Jean-Louis. À l'occasion du centenaire des premières recherches archéologiques à Saint-Bertrandde-Comminges (Lugdunum Convenarum), retour sur l'établissement chrétien du quartier du Plan. In: Comptes rendus des séances de l'Académie des Inscriptions et Belles-Lettres, 154e année, N. 3, 2010. pp. 1265-1302;

doi : $10.3406 /$ crai.2010.93011

http://www.persee.fr/doc/crai_0065-0536_2010_num_154_3_93011

Document généré le 05/06/2016 


\title{
COMMUNICATION
}

\author{
À L'OCCASION DU CENTENAIRE \\ DES PREMIÈRES RECHERCHES ARCHÉOLOGIQUES \\ À SAINT-BERTRAND-DE-COMMINGES (LUGDUNUM CONVENARUM), \\ RETOUR SUR L'ÉTABLISSEMENT CHRÉTIEN DU QUARTIER DU PLAN, \\ PAR MM. JEAN GUYON ET JEAN-LOUIS PAILLET
}

Voici cent ans, Raymond Lizop publiait dans la Revue des études anciennes un article qui a signé la naissance d'études archéologiques, au sens moderne de ce terme, sur le site de Saint-Bertrandde-Comminges qui n'avait guère attiré jusqu'alors que la curiosité des antiquaires ${ }^{1}$. Quel meilleur moyen de célébrer cet anniversaire passé assez inaperçu, sinon de revenir sur la basilique chrétienne du quartier du Plan dont le même Raymond Lizop fut l'inventeur trois ans plus tard ? Cela d'autant qu'il s'agit du premier monument à avoir été intégralement fouillé et présenté au public à Saint-Bertrand et que l'histoire de sa recherche se confond avec celle des recherches archéologiques sur ce site.

\section{Cent ans de recherches archéologiques}

Parce qu'il constituait un « inventaire des ensembles connus et des terrains susceptibles d'être fouillés méthodiquement $»^{2}$, l'article de Raymond Lizop avait valeur de programme. Un programme auquel il a su intéresser le secrétaire général de la Société française des fouilles archéologiques, Marcel Dieulafoy : dès 1912, ce dernier faisait paraître dans la Dépêche $d u$ Midi un article pour signifier son souhait de voir conduire des recherches à Saint-Bertrand ${ }^{3}$. Une telle publicité donnée à la nouvelle a aussitôt incité les habitants du lieu à défoncer leurs champs dans l'espoir d'y trouver des vestiges

1. R. Lizop, "Quelques recherches sur les ruines de Lugdunum Convenarum », Revue des études anciennes XII, 1910, p. 399-410.

2. P. Lavedan, R. Lizop, B. Sapène, « 1920-1929. Les fouilles de Saint-Bertrand-deComminges (Lugdunum Convenarum) ", Mémoires de la Société archéologique du Midi de la France XVII, 1930, p. 61.

3. M. Dieulafoy, « Pour l'Histoire », La Dépêche du Midi, 14édition, nº du 7 août 1912. 


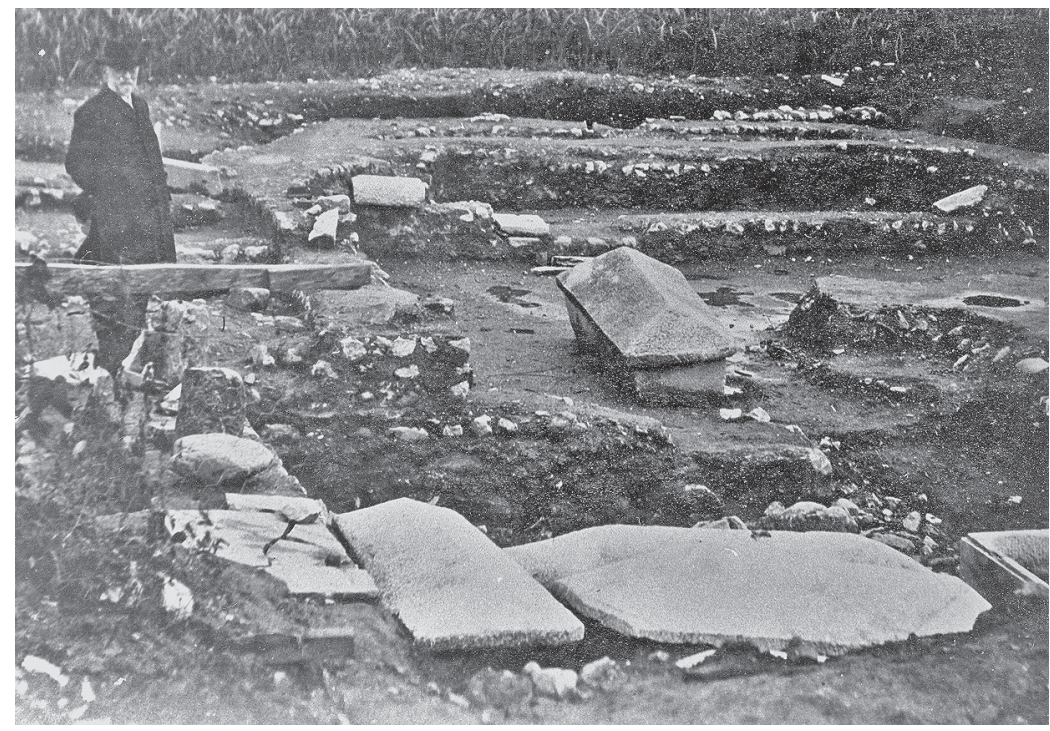

FIG. 1. - Marcel Dieulafoy en visite sur la fouille de la basilique chrétienne de Saint-Bertrand-de-Comminges, sans doute le 13 septembre 1913 (derrière lui, l'abside de l'édifice de culte; au premier plan, la nef), d'après R. Lizop, Histoire de deux cités gallo-romaines. Les Conuenae et les Consoranni (Comminges et Couserans), pl. XVII, fig. 1 (cliché J. Dieulafoy).

susceptibles de conduire à leur location aux fins de fouilles. C'est ainsi qu'en février ou mars $1913^{4}$, Alexandre Guiard découvrit deux sarcophages sur un terrain proche du cimetière du Plan. Dès qu'il eut connaissance de la chose, Raymond Lizop présenta un rapport à la Société des fouilles et sut la convaincre de lui confier une enquête sur ce site où la poursuite des recherches ne permettait pas de douter que les cuves relevaient d'un édifice de culte chrétien. Le contrat avec le propriétaire fut signé le 30 juillet et, dès le 2 août, il put entreprendre un mois et demi de fouilles plus ordonnées dont le rapport, du fait de la guerre, n'a été publié que neuf ans plus tard ${ }^{5}$. Entre temps cependant, votre confrère Marcel Dieulafoy, fort du patronage scientifique qu'il avait accordé aux fouilles (fig. 1), en avait présenté les résultats

4. Février, comme l'écrit R. Lizop, « Rapport sur les fouilles de Saint-Bertrand-deComminges (découverte d'une basilique chrétienne) ", Bulletin de la Société française des fouilles archéologiques IV, 1914-1922, p. 29, ou le 22 mars à en croire La Dépêche du Midi du 2 avril 1913 ?

5. R. Lizop, op. cit. (n. 4), p. 29-61. 
à votre compagnie lors de sa séance du 30 janvier $1914^{6}$. De ce fait, c'est à votre Académie que la basilique doit d'avoir été connue du monde savant dès les lendemains de sa découverte.

Elle n'avait pourtant été reconnue que partiellement en 1913 et ne fut entièrement dégagée qu'à l'issue de deux autres campagnes de fouilles menées en 1920 et 1926, dont la première fut conduite à nouveau par Raymond Lizop ${ }^{7}$ et l'autre conjointement par Raymond Lizop et Bertrand Sapène ${ }^{8}$. Dès lors présentée au public, elle n'a pas tardé à entrer dans les manuels, guides et ouvrages de synthèse qui se sont bornés à résumer les rapports de fouille ou le chapitre que Raymond Lizop lui avait consacré en 1931 dans sa thèse ${ }^{9}$ et à reproduire le plan qui illustrait ces publications (fig. 2). Point de réexamen critique en effet en tout cela, mais seulement de menues réserves sur l'interprétation iconographique de tel élément du mobilier funéraire ${ }^{10}$, sur la chronologie de l'édifice ${ }^{11}$ ou son évolution que Bertrand Sapène et à sa suite Robert Gavelle ont su deviner plus complexe que ne le pensaient ses inventeurs ${ }^{12}$.

Les recherches que ce même Bertrand Sapène avaient conduites entre 1927 et 1938 aux abords de la basilique pouvaient pourtant autoriser des révisions plus substantielles mais, pris par d'autres urgences, le fouilleur, qui avait en charge l'ensemble des recherches

6. M. Dieulafoy, «Basilique constantinienne de Lugdunum Convenarum », Comptes rendus des séances de l'Académie des Inscriptions et Belles-Lettres, 1914, p. 59-90.

7. R. Lizop, « Second rapport sur les fouilles de Saint-Bertrand-de-Comminges. Année 1920 », Bulletin de la Société française des fouilles archéologiques IV, 1914-1922, p. 241-249.

8. P. Lavedan, R. Lizop, B. Sapène, op. cit. (n. 2), p. 98-104.

9. R. Lizop, Histoire de deux cités gallo-romaines. Les Conuenae et les Consoranni (Comminges et Couserans), Paris-Toulouse, 1931, p. 426-438.

10. H. Leclercq, «Lugdunum Convenarum », dans Dictionnaire d'archéologie chrétienne et de liturgie, F. Cabrol, H. Leclercq (éd.), t. IX, Paris, 1930, col. 2680-2688 et spécialement col 26852686 pour l'analyse de l'étude de Marcel Dieulafoy sur le décor du sarcophage d'Aemiliana qui recèle une des rosseries les plus féroces de ce dictionnaire qui n'en manque pas : « Il fallait toute la sereine inhabitude des choses d'archéologie chrétienne pour assurer que "ces dispositions (...) font de ce chrisme un des plus précieux et peut-être le plus élégant parmi ceux qui sont connus". Et voilà l'Académie bien renseignée. Mais ce n'est pas tout, ce monogramme "fleure un parfum syrien très prononcé" qui embaumera à tout jamais l'odorat de Marcel Dieulafoy et de ses confrères. »

11. J. Hubert, L'architecture religieuse du Haut Moyen Age en France, Paris, 1952, p. 60 : «Il y a tout lieu de croire que (la basilique) ne fut édifiée qu'après l'invasion des Vandales de l'année 409 ou environ » et non à époque constantinienne comme le supposaient Marcel Dieulafoy et Raymond Lizop.

12. B. Sapène, Saint-Bertrand-de-Comminges (Lugdunum Convenarum) - Centre touristique d'art et d'histoire, Toulouse, 1961, p. 109 où est noté sur le « Plan visite» de la basilique, à hauteur du chevet : "Ce chevet renforcé ne se soude pas au chœur : un mur transversal, indépendant, les sépare. Le chevet à 3 pans serait-il postérieur (Important) »; R. Gavelle, " Pour l'histoire de Convenae : quelques repères », Revue de Comminges 75-1, 1962, p. 6 : «Le redoublement des trois pans terminaux de l'abside et divers détails indiqueraient (...) des remaniements et réemplois ", avec en note un renvoi à l'ouvrage de Bertrand Sapène. 


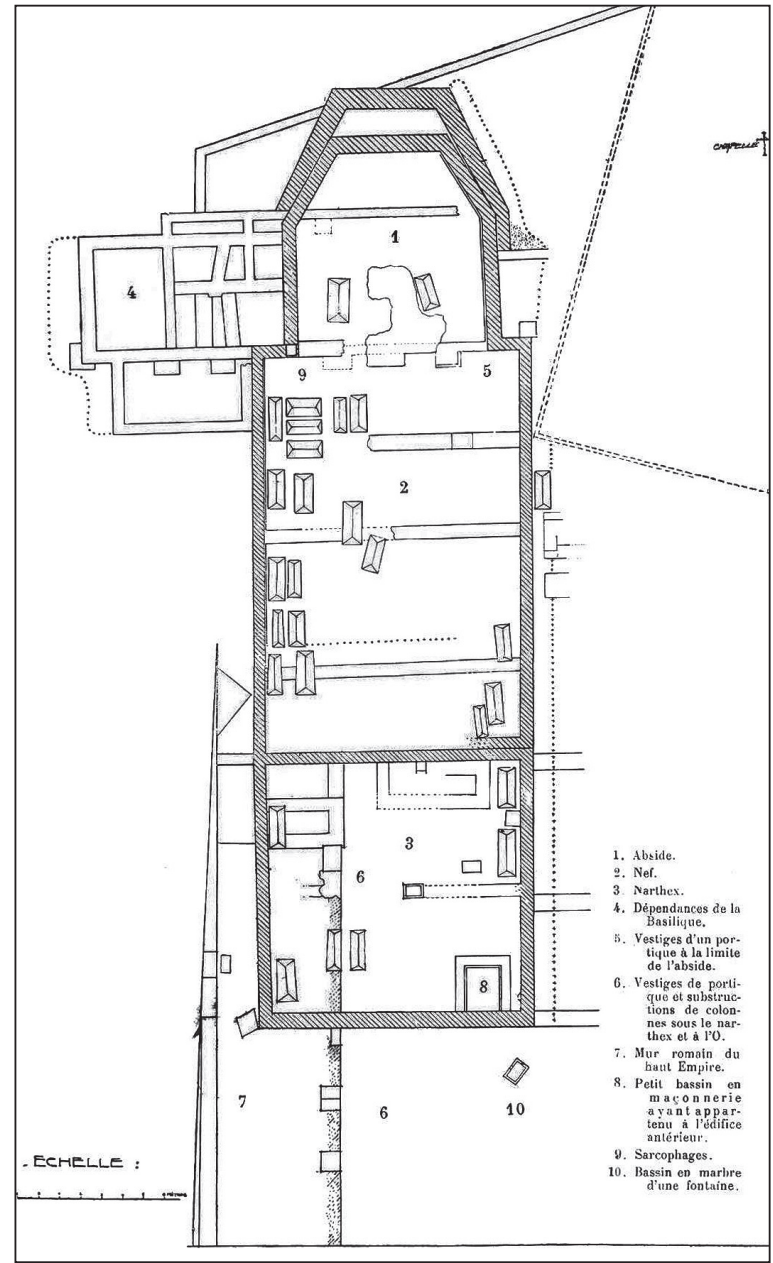

FIG. 2. - Plan de la basilique après les fouilles de 1913 à 1926, d'après R. Lizop, Histoire de deux cités gallo-romaines. Les Conuenae et les Consoranni (Comminges et Couserans), pl. XV (plan de « M. Martin, architecte à Tarbes»). 
de Saint-Bertrand, s'en est tenu à les publier ${ }^{13}$ et à attirer d'un mot l'attention sur elles dans son guide du site ${ }^{14}$. Cela suffirait à justifier que nous revenions sur ce monument, mais il est une autre raison pour qu'il fasse l'objet, quelque cent ans après son premier signalement par Marcel Dieulafoy, d'une autre communication à votre Académie : les nouvelles recherches qu'il a connues après que l'État eut racheté en 1985 à la Société archéologique du Midi de la France les terrains qu'elle avait acquis à l'issue des fouilles.

Le ministère de la Culture a souhaité en effet que les vestiges qu'ils recelaient fussent l'objet de fouilles complémentaires avant d'être restaurés et convenablement présentés au public. Il a donc suscité la création d'un projet collectif de recherche qui a mobilisé les efforts de plusieurs équipes pendant une décennie et demie ; cela afin de réexaminer les éléments déjà fouillés, mais aussi de conduire de nouvelles investigations, sur le rempart de la ville haute ou, dans la plaine, sur le site de Coupéré. De là, s'agissant de la basilique chrétienne, l'enquête que nous avons pu mener de 1985 à 1992 sur l'édifice et ses abords, avec la collaboration avec Christian Landes et le concours de jeunes collègues ou d'étudiants avancés comme Christine Delaplace et Marc Griesheimer et celui de bénévoles, étudiantes et étudiants pour la plupart ${ }^{15}$.

Comme celles des autres monuments du site, ces fouilles ont vocation à être publiées dans une série $a d$ hoc de la collection des « Études d'archéologie urbaine » éditée par la Fédération Aquitania, à la suite des quatre volumes parus à ce jour dont l'ordonnance

13. Au sud de la basilique, il s'est agi d'un sondage d'extension limitée dont la publication a paru sous la signature de R. Lizop, « Rapport sur les fouilles de Saint-Bertrand-de-Comminges de 1933 à 1938, troisième partie », Mémoires de la Société archéologique du Midi de la France XXI, 1947, p. 128-129. Au nord en revanche, les recherches ont porté sur de nombreuses parcelles et mobilisé les efforts de B. Sapène pendant une dizaine d'années ; aussi leur publication est-elle également très morcelée : P. Lavedan, R. Lizop, B. Sapène, op. cit. (n. 2), p. 110-111 ; « Rapport sur les fouilles de Saint-Bertrand-de-Comminges (Lugdunum Convenarum) 1929-1930 ", ibid., XVIII, 1932, p. 8-20 ; «Les fouilles à Saint-Bertrand-de-Comminges », ibid., p. 164-175 ; R. Lizop, « Rapport sur les fouilles de Saint-Bertrand-de-Comminges de 1933 à 1938, troisième partie », ibid., XXI, 1947, p. 126-127.

14. B. Sapène, op. cit. (n. 12), p. 109, où sont signalés une « cour ou place (du vicus Florentinus?) » au sud de la basilique et une « riche maison romaine du Haut Empire avec portique (de place ou cour) » au nord.

15. Soit une campagne de reconnaissance, deux programmes triennaux de recherche pendant lesquelles les fouilles ont duré en moyenne quatre à cinq semaines en mobilisant une trentaine de bénévoles et une ultime campagne consacrée à un sondage de vérification. Ces recherches, comme les actuels travaux de préparation à la publication, ont bénéficié de l'aide constante des Conservateurs régionaux de l'archéologie successifs, R. Lequément, M.-G. Colin, M. Vidal et M. Vaginay, et de leurs agents, Chr. Dieulafait, B. Marty et D. Schaad, mais aussi du concours de J.-L. Schenck et de l'équipe du Musée archéologique départemental de Saint-Bertrand-de-Comminges. Que tous trouvent ici l'expression de nos remerciements. 
répond, au moins en gros, à un ordre chronologique ${ }^{16}$. Elles ont déjà fait l'objet de signalements sommaires et de présentations synthétiques ${ }^{17}$ qui sont autant de pierres d'attente de cette publication à laquelle nous mettons actuellement la dernière main, en compagnie de collègues en charge de l'étude du matérie ${ }^{18}$. Notre propos s'autorise naturellement de la réflexion menée en commun au sein de cette équipe, mais il bénéficiera également des acquis des recherches conduites ces dernières décennies par les autres fouilleurs du site, qui ne se limitent pas aux publications que nous avons signalées. Comme en témoignent le guide archéologique qu'ils ont élaboré collectivement ${ }^{19}$ et de nombreuses études de synthèse ${ }^{20}$, leurs enquêtes ont conduit en effet à renouveler fondamentalement l'appréhension de l'évolution urbaine du chef-lieu des Convènes, ce qui n'est pas sans incidence sur l'interprétation de la basilique comme on le verra.

Ce sont là autant de dettes qu'il nous est agréable de reconnaître, au même titre que celle que nous avons contractée auprès

16. A. Badie, R. Sablayrolles, J.-L. Schenck, Saint-Bertrand-de-Comminges I. Le temple du forum et le monument à enceinte circulaire, Bordeaux, 1994 ; P. Aupert, R. Monturet et alii, SaintBertrand-de-Comminges II. Les thermes du forum, Bordeaux, 2002 ; S. E. Cleary, J. Wood, SaintBertrand-de-Comminges III. Le rempart de l'Antiquité tardive de la ville haute, Bordeaux, 2006 ; G. Fabre, J.-L. Paillet, Saint-Bertrand-de-Comminges IV. Le macellum, Bordeaux, 2009.

17. J. Guyon, J.-L. Paillet, « Saint-Bertrand-de-Comminges, basilique de la ville basse », dans Les premiers monuments chrétiens de la France, t. II, Sud-Ouest et Centre, N. Duval (éd.), Paris, 1996, p. 177-189 ; J. Guyon, "Saint-Bertrand-de-Comminges ", dans Topographie chrétienne des cités de la Gaule des origines au milieu du VIII siècle, XIII. Province ecclésiastique d'Éauze (Novempopulana), N. Gauthier, B. Beaujard, F. Prévot (éd.), Paris, 2004, p. 76-80. ; Id., « La basilique chrétienne de Saint-Bertrand-de-Comminges, édifice singulier ou "fossile directeur"d'une évolution urbaine? ", dans Paul-Albert Février de l'Antiquité au Moyen Âge, actes du colloque de Fréjus, 7 et 8 avril 2001, M. Fixot (éd.), Aix-en-Provence, 2004, p. 201-214.

18. Les éléments d'architecture et les sarcophages sont étudiés par J.-L. Paillet ; la mosaïque par V. Blanc-Bijon ; les restes anthropologiques par É. Crubézy, S. Duchesne, Ph. Gaury, L. Morel, P. Murail et Th. Romon ; les monnaies par J.-P. Bost ; le mobilier céramique par E. Grisoni ; le verre par M.-Th. Marty ; le mobilier métallique par V. Susini ; la tabletterie par Ph. Prévot ; la faune par Y. Lignereux.

19. P. Aupert, E. Boube, Chr. Dieulafait et alii, Saint-Bertrand-de-Comminges, Collection des « Guides archéologiques de la France », n³3, Paris, 1996.

20. Dont on ne citera ici que les plus notables : J. Guyon, « From Lugdunum to Conuence : recent work on Saint-Bertrand-de-Comminges (Haute-Garonne) », Journal of Roman Archaeology 4, 1991, p. 89-122 ; J.-L. Paillet, C. Petit, « Nouvelles données sur l'urbanisme de Lugdunum des Convènes. Prospection aérienne et topographie urbaine ", Aquitania 10, 1992, p. 109-144 ; R. Sablayrolles, "Quinze années de recherches collectives à Saint-Bertrand-de-Comminges », Revue de Comminges 115-3, 1999, p. 399-415 ; J. Guyon, « De Lugdunum des Convènes à Conuenae, puis Saint-Bertrand : l'évolution urbaine de Saint-Bertrand-de-Comminges à la lumière des fouilles récentes ", dans Itinéraires de Saintes à Dougga, Mélanges offerts à Louis Maurin, J.-P. Bost, J.-M. Roddaz, F. Tassaux (éd.), Bordeaux, 2003, p. 131-149; Id., « Saint-Bertrand-de-Comminges », dans Topographie chrétienne des cités de la Gaule, op. cit. (n. 17), p. 51-81 et spécialement p. 58-74 pour l'évolution topographique ; R. Sablayrolles, A. Beyrie, Le Comminges (Haute-Garonne), Carte archéologique de la Gaule, 31/2, M. Provost (dir.), Paris, 2006, p. 248-410 et spécialement p. 254-263. 
des découvreurs de la basilique, Raymond Lizop, Marcel Dieulafoy et Bertrand Sapène, à la mémoire desquels nous souhaitons dédier cette communication. Ce n'est que justice tant nos recherches s'inscrivent dans le sillage qu'ils avaient eux-mêmes tracé. Pour autant, elles nous ont conduits vers d'autres horizons, de sorte qu'au terme de notre enquête, nous retirons du monument une " certaine idée » qui diffère sensiblement de celle à laquelle s'étaient arrêtés nos prédécesseurs.

\section{Un monument à l'évolution complexe inséré dans un dense tissu urbain}

Pour prendre la mesure de la révision à laquelle conduisent les fouilles récentes, qu'il suffise de rapprocher le plan dressé par nos devanciers au terme de leurs recherches (fig. 2) et le plan schématique interprété qui ressort de nos travaux (fig. 3). De ce rapprochement, pour faire bref, nous ne retiendrons que trois traits. D'abord que loin d'être isolée comme elle paraissait naguère, la basilique relève d'un établissement chrétien qui comptait des annexes d'une étendue au moins égale, sinon supérieure à la sienne. Ensuite que cet établissement a connu une évolution complexe que l'on pouvait seulement soupçonner sur le plan dressé après la campagne de 1929 par le doublon des appareils du chevet et l'enchevêtrement des murs des annexes nord. Enfin qu'il a été élevé sur un terrain bien plus densément occupé dans l'Antiquité que ne le signalaient nos prédécesseurs. Ces acquis tiennent aux nouveaux horizons que nous avons assignés dès 1985 à notre enquête après qu'une campagne exploratoire nous eut convaincus de la nécessité d'étendre les recherches aux abords de la basilique si nous voulions préciser la chronologie d'un monument dont nos devanciers avaient affouillé le sol jusqu'aux niveaux du Haut Empire. Cela a été possible grâce à la disponibilité des propriétaires riverains ${ }^{21}$ qui nous vaut de pouvoir replacer désormais l'édifice de culte non seulement dans le contexte d'un établissement plus vaste, mais aussi dans celui de l'îlot urbain dont il relevait.

Un îlot urbain : sur la fig. 3, la chose n'apparaît guère à vrai dire que par la présence à l'est d'une rue sur laquelle empiète

21. Au sud de la basilique, il s'agit de MM. Huchan et Marot, à l'est de M. Grégoire, au nord de M. Trey et à l'ouest de M. Escoubas; qu'ils trouvent ici le témoignage de notre reconnaissance. 


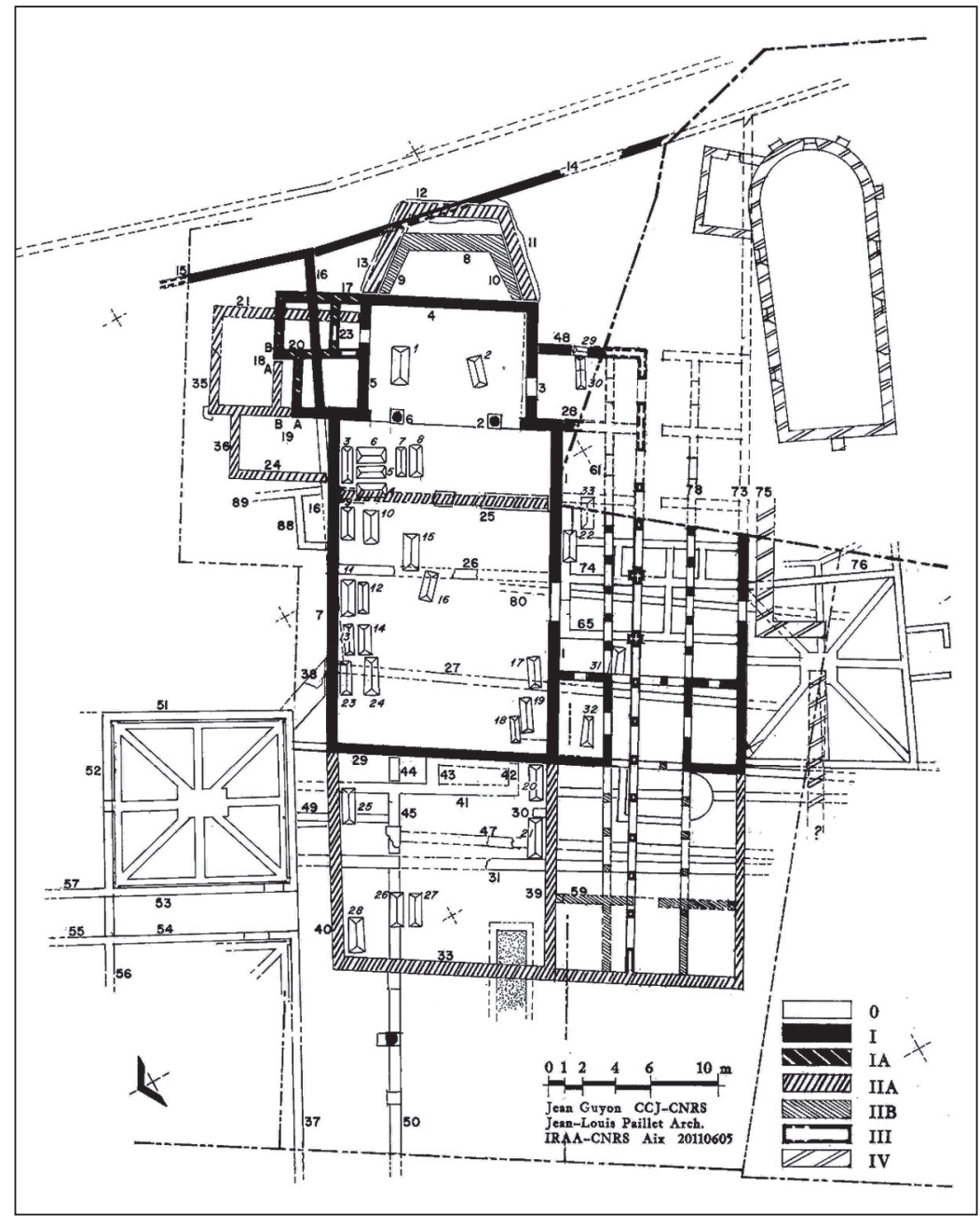

FIG. 3. - Plan évolutif de l'établissement chrétien du quartier du Plan à l'issue des recherches de 1985 à 1992 : 0 : éléments antérieurs à l'établissements ; I, I A, II A, II B et III : états successifs de l'établissement ; IV : éléments postérieurs à l'établissement : la chapelle romane Saint-Julien et un possible rempart de la ville basse (dessin J.-L. Paillet).

modérément le chevet de la basilique et des éléments de maisons au nord et au sud. Pour la mettre en évidence, un changement d'échelle s'impose, qui conduit à considérer dans son ensemble le plan d'urbanisme de Lugdunum Convenarum tel qu'il ressort des recherches récentes, en particulier celles de Jean-Louis Paillet et 
Catherine Petit ${ }^{22}$. L'origine de ce plan, selon ces chercheurs, tient à un carrefour de pistes disposées en étoile, lieu probable à époque protohistorique d'un marché - sans doute un forum boarium (fig. 4). Les pistes, à peine rectifiées, ont servi en effet d'ossature au réseau des rues de la ville romaine dont elles expliquent l'irrégularité tandis que le carrefour est demeuré l'épicentre de la cité, au point d'avoir été matérialisé à époque tibérienne par l'érection d'un monument commémoratif - une colonne ? - comme Jean-Luc Schenck l'a bien montré23. Il signalait ainsi le cœur de l'espace public dont l'ordonnance, pour reprendre une expression de Pierre Aupert ${ }^{24}$, est réglée par une « quadripartition » qui tient à la succession, d'est en ouest, d'une basilique qui reste à retrouver, du forum, du temple du culte impérial, de la place qui avait succédé à l'ancien foirail enfin (fig. 5).

Attachons-nous à cette place dans laquelle Jean-Louis Paillet et Catherine Petit ont reconnu la « plaza Mayor» de la ville antique ${ }^{25}$. Le jugement se justifie par sa situation au croisement du cardo et du decumanus maximus comme par l'importance et la qualité des monuments qu'elle recèle ${ }^{26}$ ou qui en constituent l'écrin : au sud-est, un arc tétrapyle sur le decumanus qui marque son débouché sur la place ; le temple du culte impérial et de vastes thermes à l'ouest ; un macellum au nord, qui est le plus important des Gaules ${ }^{27}$. Les autres rives sont en revanche dévolues à un usage privé : des boutiques, sans doute, à l'est et au sud un îlot d'habitations - celui au sein duquel a été ultérieurement établie la basilique (fig. 6).

De cet îlot de forme trapézoïdale, trois des rues riveraines sont connues avec certitude ; la dernière, au sud, a été restituée par hypothèse dans le prolongement d'une rue identifiée plus à l'ouest, à hauteur de la porticus post scaenam du théâtre ${ }^{28}$ (fig. 5). La moitié occidentale de l'îlot a été largement reconnue par Bertrand Sapène lors des fouilles qu'il a conduites de 1927 à 1931 au nord de la

22. J.-L. Paillet, C. Petit, op. cit. (n. 20), p. 109-144.

23. A. Badie, R. Sablayrolles, J.-L. Schenck, op. cit. (n. 16), p. 121-162.

24. P. Aupert, R. Monturet et alii, op. cit. (n. 16), p. 104.

25. J.-L. Paillet, C. Petit, op. cit. (n. 20), p. 121.

26. Il s'agit du monument commémoratif déjà signalé et d'un autre monument quadrangulaire plus au nord, sur l'interprétation duquel B. Sapène, «Contribution à l'urbanisme de Lugdunum Convenarum. Le carrefour du temple (à l'est du forum) », Mémoires de la Société archéologique du Midi de la France XXIV, 1956, p. 17-32, avait hésité. Sans doute sa première intuition - reconnaître en lui un sanctuaire de carrefour - était-elle la meilleure, comme le suggère J.-L. Schenck (A. Badie, R. Sablayrolles, J.-L. Schenck, op. cit. [n. 16], p. 126).

27. Pour l'arc, on renverra à J.-L. Paillet, C. Petit, op. cit. (n. 20), p. 121 et 135, rubrique D 1 ; pour les autres monuments publics, aux publications citées supra n. 16 .

28. J.-L. Paillet, C. Petit, op. cit. (n. 20), p. 135, rubrique D 1. 


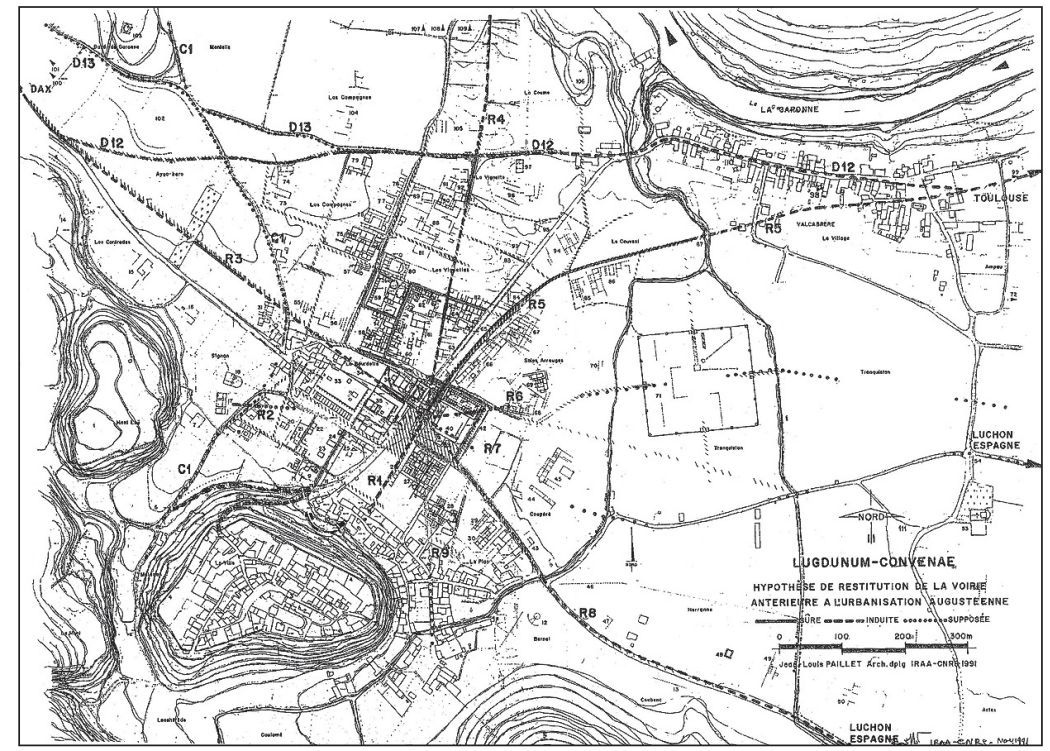

FIG. 4. - Le réseau des voies desservant le site de Lugdunum Convenarum avant l'urbanisation de la cité à époque augustéenne, d'après C. Petit et J.-L. Paillet (dessin J.-L. Paillet).

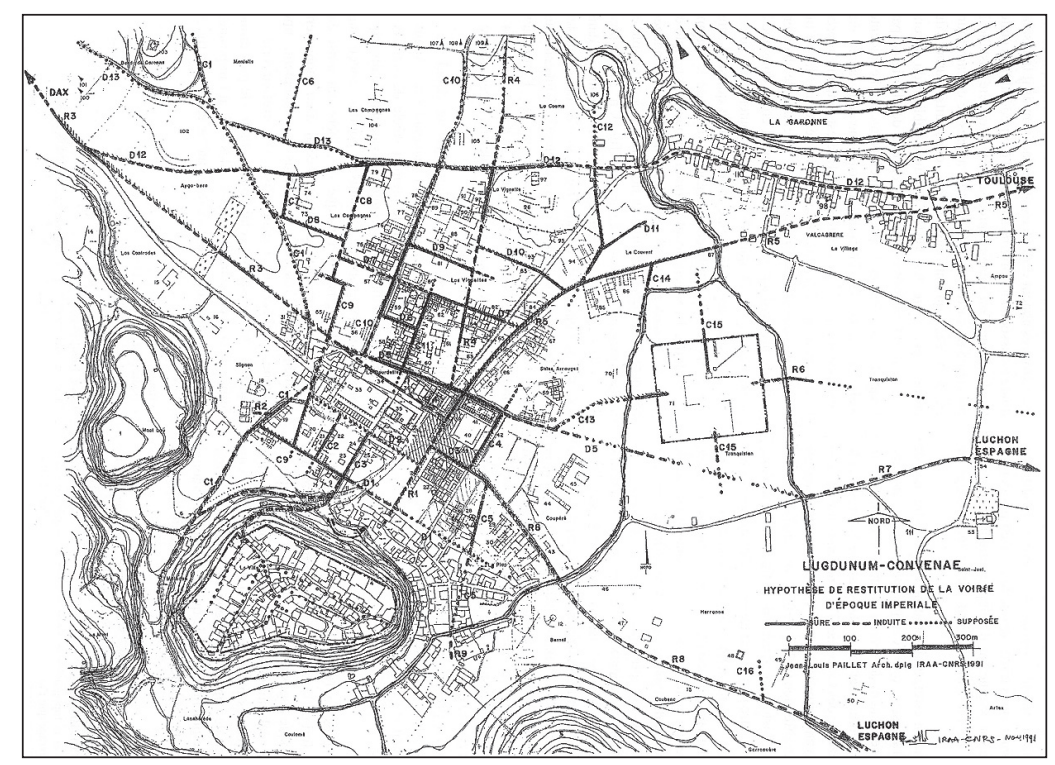

FIG. 5. - Le plan d'urbanisme de Lugdunum Convenarum à époque augustéenne (dessin J.-L. Paillet). 


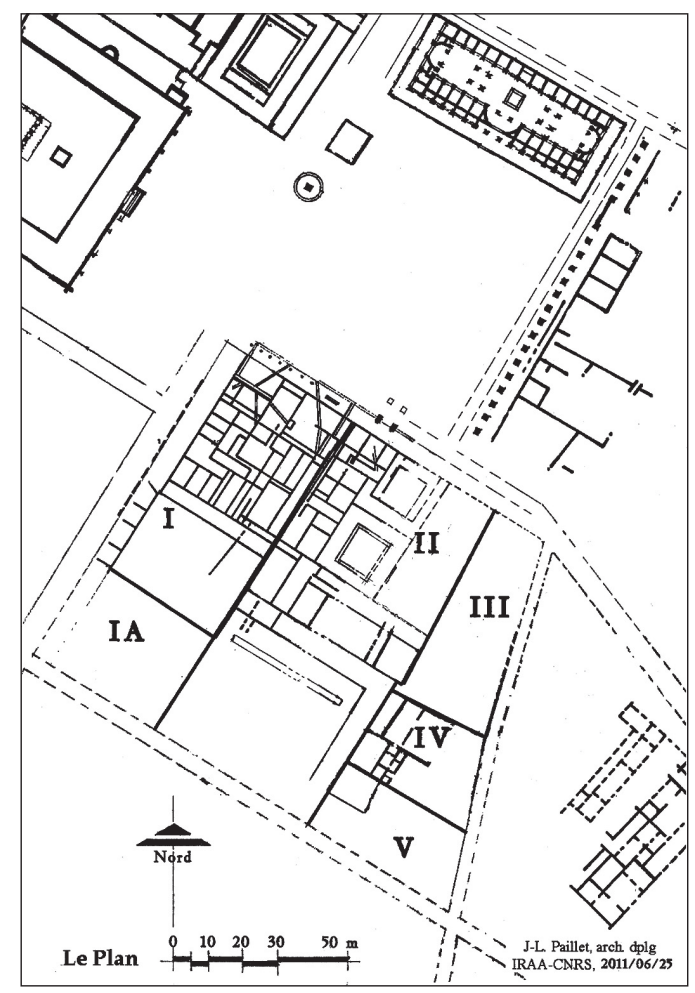

FIG. 6. - L'îlot urbain situé au sud du centre monumental de Lugdunum Convenarum $\mathrm{au} \mathrm{I}^{\mathrm{er}}$ siècle de notre ère (dessin J.-L. Paillet).

basilique $^{29}$. Mais là où notre prédécesseur n'avait identifié qu'une seule habitation, la présence d'un ambitus commande de reconnaître à coup sûr deux domus, I et II, et sans doute une troisième, tant l'étirement de la parcelle occidentale rend probable l'existence au sud de la domus I d'une autre domus, I A. Il n'en va pas de même pour la parcelle centrale assurément dévolue à la seule domus II, d'une ampleur exceptionnelle pour Lugdunum Convenarum : près de $5500 \mathrm{~m}^{2}$, presque équitablement partagés entre le corps d'habitation et le jardin sur lequel il ouvre au midi ${ }^{30}$. De la moitié orientale de l'îlot n'est connu en revanche - et assez partiellement - que le quadrant sud-est. Il compte deux parcelles dont l'une, V, est occupée

29. On en trouvera les références supra n. 13.

30. Dont on ignore cependant s'il comptait au sud la dernière aile d'un quadriportique ou un autre corps d'habitation. 
par une autre domus ${ }^{31}$ tandis qu'au nord, en IV, nos recherches ont mis en évidence de petits thermes dont on ne sait s'ils ont une existence autonome ou s'ils dépendent d'une domus voisine. La parcelle III, au nord-est, reste largement terra incognita ${ }^{32}$.

Malgré cette lacune et celle qui lui fait pendant au sud-ouest, la qualité et l'importance des éléments qui ont été reconnus ne permettent pas de douter que l'îlot était un des plus huppés de la cité du Haut Empire, ce qui s'explique assez si l'on songe à sa situation. Or l'important pour notre propos est qu'il n'avait en rien perdu ce statut à l'aube de l'Antiquité tardive, bien au contraire (fig. 7). Toutes les transformations que l'on soupçonne ou qu'on a pu vérifier au sein des domus sont allées en effet dans le sens d'un embellissement et d'un accroissement du confort, sauf pour la domus I dont la superficie a été légèrement amputée au profit de la domus II dont nous avons dit pourtant l'ampleur remarquable. Cela afin d'aménager dans le quadrant sud-ouest du corps d'habitation une grande exèdre et des thermes privés que Bertrand Sapène a mis en évidence sans pouvoir indiquer la date de ces transformations ${ }^{33}$. Nous les associerions volontiers à d'autres travaux que nous avons pu dater du courant du IV ${ }^{\mathrm{e}}$ siècle par notre propre fouille de l'angle sud-est de la maison où deux salles ont été dotées d'un système de chauffage par conduits rayonnants installés au-dessus du sol originel et pour l'une d'elles, d'un revêtement de sol en mosaïque polychrome décoré d'un motif de nattes ${ }^{34}$. Sans qu'ils atteignent un tel luxe, des travaux analogues ont porté dans le même temps sur l'unique salle connue à ce jour de la domus $\mathrm{V}$, réaménagée elle aussi en salle chauffée. Ces retouches sont cependant mineures au regard des bouleversements qui ont affecté la parcelle IV où le tissu assez lâche des thermes et de leurs annexes a fait place à des salles au sol en béton dont il nous a été malaisé de décider si elles relèvent d'une domus indépendante ou d'une extension vers le nord de la domus $\mathrm{V}^{35}$.

31. Dont n'est connue qu'une salle de taille équivalente à celle des salles les plus importantes de la domus II.

32. À l'exception de ses franges méridionales sur lesquelles nous avons pu ouvrir un sondage d'extension très limitée qui n'a pas livré de résultats significatifs.

33. Pour l'exèdre, consulter « Les fouilles à Saint-Bertrand-de-Comminges », op. cit. (n. 13), p. 166-167 et pour les thermes P. Lavedan, R. Lizop, B. Sapène, op. cit. (n. 2), p. 110-111.

34. Qui peut cependant n'avoir été installé que dans un second temps.

35. Nul doute en revanche qu'il s'agit d'une demeure de même qualité que les domus avoisinantes, l'unique salle qui en a sûrement été reconnue sous les annexes de la basilique mesurant une centaine de mètres carrés de superficie. 


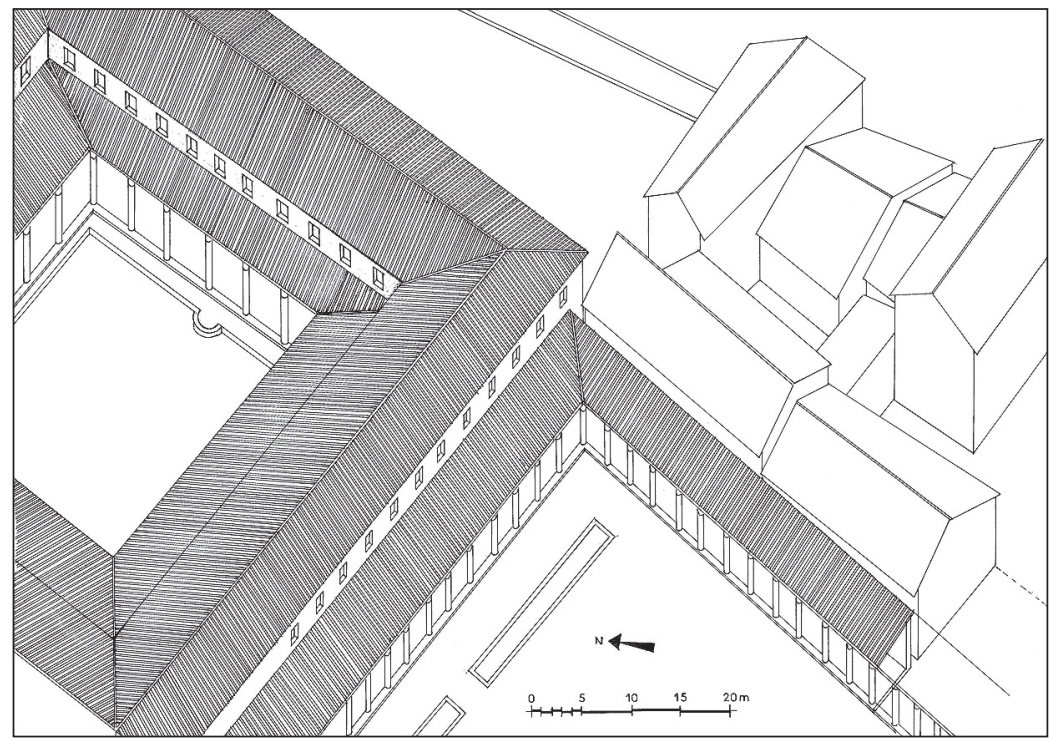

FIG. 7. - Axonométrie du quadrant sud-est de l'îlot au sud du centre monumental de Lugdunum Convenarum dans l'état antérieur à la construction de l'établissement chrétien : au premier plan, la domus II ; à l'arrière-plan, évocation des domus IV et $\mathrm{V}$ beaucoup plus mal connues (dessin M.-C. Monguillan).

Peu importe cette incertitude, le principal étant de noter que l'évolution de l'îlot a indéniablement conduit non à un dépérissement, mais à une densification de l'habitat sur l'ensemble de ses parcelles qu'il est donné de connaître. Cela n'a rien pour étonner quand un semblable phénomène se vérifie sur la place publique qui le jouxte au nord : d'espace ouvert qu'elle était depuis les origines, elle a mué, au $\mathrm{IV}^{\mathrm{e}}$ siècle sans doute elle aussi, en un quadriportique flanqué au nord par une basilique construite en lieu et place de l'ancien macellum et doté au sud d'une salle axiale rectangulaire, temple ou curie. Sans doute faut-il reconnaître là, comme Georges Fabre et Jean-Louis Paillet l'ont récemment proposé, un forum tripartite ${ }^{36}$. Or c'est dans ce contexte urbain à la fois très riche et très dense qu'a été implanté l'établissement chrétien (fig. 8). 


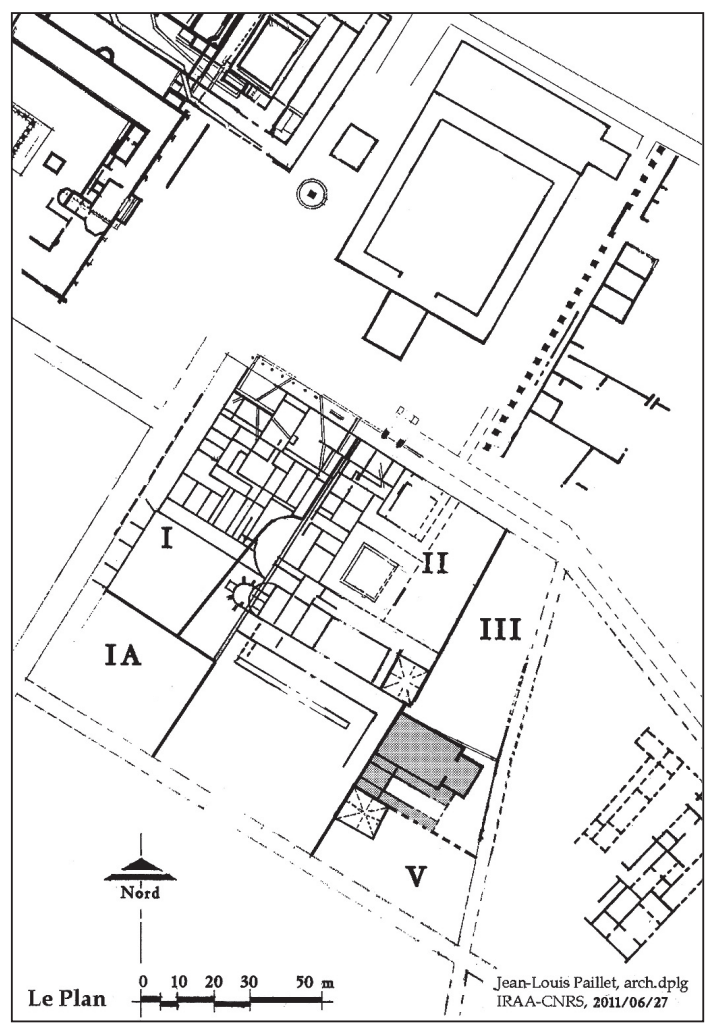

FIG. 8. - L'îlot urbain situé au sud du centre monumental de Lugdunum Convenarum $\mathrm{au} \mathrm{V}^{\mathrm{e}}$ siècle de notre ère (dessin J.-L. Paillet).

\section{Le premier établissement chrétien}

Comme le montre la fig. 8, les contours de l'établissement chrétien épousent ceux de la parcelle IV de l'îlot, sauf au nord où le mur gouttereau de la basilique chevauche en oblique le mur mitoyen de la parcelle III. La rectification du tracé est si minime qu'elle incline à penser que les autres empiètements sur cette parcelle qui tiennent à des salles annexes greffées sur le chœur de la basilique participent, non de l'état originel de l'établissement, mais des extensions qu'il a connues. Si tel est bien le cas, son insertion au sein de l'îlot aurait été la plus discrète qui soit. 


\section{L'ORDONNANCE DU PLAN}

Un mur de direction est-ouest qui divisait précédemment la domus établie sur la parcelle IV en deux parts sensiblement égales a été mis à profit pour distribuer les espaces au sein de l'établissement chrétien. Il a suffi de prendre appui sur lui ou mieux, sans doute, de le conserver en élévation et de le prolonger vers l'est pour marquer entre la basilique au nord et ses annexes au sud la césure majeure d'un nouveau programme architectural (fig. 9).

\section{La basilique}

Au regard du plan dressé par nos prédécesseurs (fig. 1), la basilique, telle qu'elle était à l'origine, peut sembler modeste. C'est qu'il faut négliger deux éléments de ce plan. D'abord le chevet (ou plutôt les deux chevets successifs à pans coupés) dont nos devanciers avaient soupçonné de longue date qu'ils relevaient d'une adjonction $^{37}$ - chose qu'a suffi à confirmer le nettoyage de l'édifice en mettant en évidence le collage de leurs appareils contre celui de la basilique dont tous les éléments sont en revanche chaînés. Ensuite l'extrémité occidentale du monument que Robert Lizop avait interprétée comme un narthex ${ }^{38}$ et qu'il faut tenir elle aussi comme une adjonction, ne fût-ce que parce que son mur nord présente une direction oblique par rapport à celle du reste du mur gouttereau, ce qui serait difficilement concevable pour une construction d'une seule venue ${ }^{39}$.

La basilique primitive apparaît dès lors comme un bâtiment qui mesure 26,75 m hors œuvre d'est en ouest pour une largeur de $13,50 \mathrm{~m}$ à 1 'ouest et de 10,25 m à l'est. Son plan est des plus simples et des plus communs - un vaisseau prolongé à l'est par un chœur à chevet plat - mais ses proportions sont harmonieuses. Le rapport entre ces deux éléments est sensiblement de 3 à 1 en effet et leurs rectangles juxtaposés présentent d'autre part un même rapport de $3 / 2$ environ entre leur longueur et leur largeur. Si le chœur paraît cependant assez trapu, cela tient au fait que son grand axe est perpendiculaire à celui du vaisseau. En raison de son enclavement au sein de

37. Voir supra n. 12 les remarques de Bertrand Sapène et Robert Gavelle, qui reprenaient d'ailleurs par là à leur compte une intuition de Marcel Dieulafoy, op. cit. (n. 6), p. 71 .

38. R. Lizop, op. cit. (n. 7), p. 243-244.

39. Sans compter que ses fondations présentent des remplois d'éléments d'architecture qui ne se rencontrent nulle part ailleurs dans la basilique et d'autres arguments que l'on trouvera infra p. 1295. 


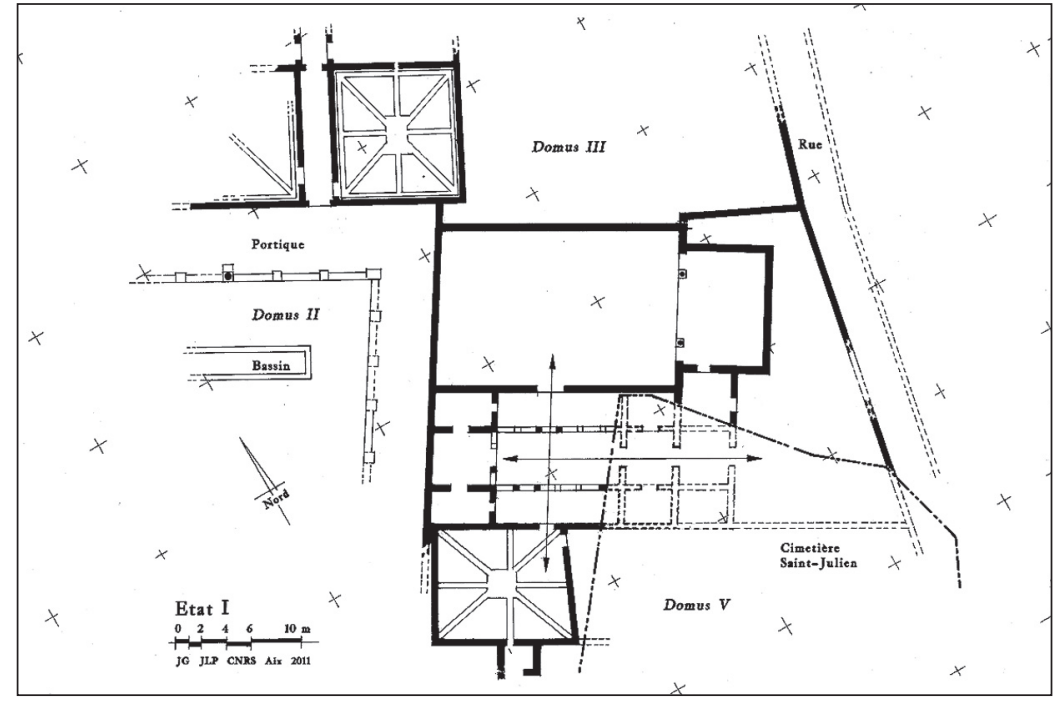

FIG. 9. - L'état I de l'établissement chrétien (dessin J.-L. Paillet).

l'îlot, le monument ne pouvait ouvrir que vers le sud par une porte dont ne subsiste aucune trace mais qui était certainement située à peu près à mi-longueur du mur gouttereau méridional ${ }^{40}$.

\section{Les annexes méridionales}

Nul doute que les annexes méridionales de la basilique s'étendaient continûment depuis le chœur de l'édifice jusqu'au droit de sa façade occidentale. Cette continuité est masquée aujourd'hui par le cimetière de Saint-Bertrand dont l'angle nord-ouest s'enfonce comme un coin au sein de l'établissement chrétien, ce qui conduit à n'avoir des annexes qu'une vue partielle.

Cela vaut surtout à l'est où ne sont connus que deux murs accolés au chœur et à l'épaulement de la basilique qui délimitent un espace de 4 m environ d'est en ouest et au moins autant du nord au sud. Un cliché de Jeanne Dieulafoy pris à l'issue de la campagne de 1913 paraît garder trace du seuil qui mettait en communication cet espace avec le chœur. Une autre porte existait probablement à l'est car le sarcophage installé dans l'appareil du mur oriental que nos fouilles

40. Le fait se déduit de la présence, dans le portique du dernier état des annexes méridionales, d'un seuil monumental qui commandait également à n'en pas douter l'accès à la basilique dont il signe ainsi l'emplacement. 
ont mis en évidence doit sans doute cette singulière disposition au fait qu'il avait été placé sous un seuil.

À l'ouest en revanche, les annexes ont pu être reconnues sur une surface de près de $150 \mathrm{~m}^{2}$ qui répond pour l'essentiel à une salle de la domus antérieure à la basilique dont trois des murs ont été conservés : au sud et à l'ouest, les murs mitoyens des parcelles $\mathrm{V}$ et II ; au nord, le mur de refend de la domus remployé comme mur gouttereau de la basilique. Au sein de cette enveloppe, nos recherches ont livré un carroyage formé par un mur transversal et deux murs longitudinaux qui délimitent une bande centrale de quelque 4,25 m de largeur dans œuvre et deux secteurs latéraux larges de 2,50 m au nord comme au sud.

De telles dispositions pourraient répondre indifféremment à l'extrémité occidentale d'un édifice à trois nefs précédé à l'ouest par une sorte de vestibule triparti ou à un espace ouvert entouré par trois corps de bâtiment. Mais l'hésitation n'est pas permise si l'on prend en compte la médiocrité des fondations des murs ${ }^{41}$ qui interdit de penser qu'ils aient pu supporter une élévation importante. On privilégiera donc sans hésiter la seconde restitution : une cour flanquée à l'ouest par trois salles et bordée au nord et au sud par des portiques adossés à la basilique et à la domus $\mathrm{V}$ avec lesquelles ils étaient en communication. La chose, certaine pour la basilique ${ }^{42}$, est seulement probable pour la domus $\mathrm{V}$, mais la présomption qu'elle ait ouvert sur le portique est d'autant plus forte qu'à retenir cette hypothèse ${ }^{43}$, l'accès aux deux bâtiments serait disposé sur un même axe perpendiculaire à la cour (fig. 9).

\section{UN ÉTABLISSEMENT MONUMENTAL}

Le plan de l'établissement chrétien suffit à convaincre de quel programme architectural très réfléchi il procède, mais pour mesurer la dimension proprement monumentale que ce programme a revêtue, il convient d'entrer plus avant dans les restitutions.

41. Qui n'a pu être vérifiée cependant qu'en un point, où elle atteint $0,30 \mathrm{~m}$ à peine. Mais les recherches plus approfondies sur les extensions des annexes vers l'ouest que l'on examinera d'ici peu ont conduit à des résultats analogues, de sorte que l'on ne doutera guère que cette cote vaut pour l'ensemble des murs des annexes primitives.

42. Voir supra n. 40 .

43. Qui tient principalement à la présence contre le mur méridional du portique de deux blocs de marbre d'une longueur totale de 1,60 m insérés dans le sol en béton de tuileau, qui ont pu servir d'appui à une marche spoliée donnant accès à un seuil disparu. Cela par analogie avec la situation que nous avons vérifiée dans la domus II voisine où un tel dispositif était intégralement conservé. 


\section{La basilique}

Proposer une restitution de la basilique conduit à achever le travail de déconstruction des hypothèses qui avaient été présentées par les premiers fouilleurs du site, et singulièrement celles de Marcel Dieulafoy dont la légitimité a progressivement été révoquée en doute au fil des recherches ultérieures.

Cela vaut d'abord pour le plan de l'édifice qu'il tenait pour un monument à trois nefs - une nef centrale et deux collatéraux plus modestes - dont il s'est efforcé de restituer le module de construction, précisément fondé selon lui sur un pied romain de $0,2958524 \mathrm{~m}^{44}$ hérité du remploi d'un monument antérieur dont il avait cru à tort reconnaître l'existence à hauteur de l'arc de chœur ${ }^{45}$. C'était là déployer trop d'ingéniosité et ses successeurs ont préféré passer sous silence cette métrologie comme ils ont abandonné dès 1922 le plan restitué d'une basilique à trois nefs qui lui servait de prétexte $^{46}$. Cela parce que leurs fouilles n'avaient mis au jour nulle trace de fondations d'un stylobate ou de bases au sein d'un édifice dont la largeur n'est pas telle en outre qu'elle implique l'existence de nefs multiples ${ }^{47}$. On s'en tiendra donc comme eux à un bâtiment à nef unique pour la couverture duquel les hautes futaies des Pyrénées étaient certainement aptes à fournir l'approvisionnement nécessaire.

Une semblable révision s'impose à propos des élévations que Marcel Dieulafoy imaginait très modestes. En témoigne la coupe transversale restituée de la basilique qu'il a personnellement dressée en imaginant assez curieusement une unique charpente à deux pans pour couvrir la nef centrale et les prétendus collatéraux (fig. 10) ${ }^{48}$. Nul doute aujourd'hui qu'une telle restitution est invraisemblable à cause de l'existence assurée d'un portique au sud de la basilique :

44. M. Dieulafoy, op. cit. (n. 6), p. 79-83.

45. Cela à cause de la présence de forts blocs en remploi dans les fondations de cet arc et des épaulements de la nef, qui répondent en fait à des chaînages d'angle présents dans l'ensemble de l'édifice.

46. M. Dieulafoy, op. cit. (n. 6), fig. 1, p. 62 que R. Lizop a reprise telle quelle dans son premier rapport, op. cit. (n. 4), fig. 3, p. 35, avant de lui substituer dans son second rapport, op. cit. (n. 7), la fig. 37, p. 243, sur laquelle la basilique ne compte qu'une nef.

47. La longueur des fermes supportant la charpente serait en effet de 13 à $14 \mathrm{~m}$, ce qui est inférieur aux dimensions relevées, par exemple, pour deux édifices à peu près contemporains, SainteSabine, où la nef centrale est large de $14,30 \mathrm{~m}$, ou a fortiori Sainte-Marie-Majeure où elle atteint près de $18 \mathrm{~m}$ : cf. R. Krautheimer, W. Frank1, S. Corbett, Corpus basilicarum christianarum Romae, III, Cité du Vatican-New York, 1971, p. 22 pour Sainte-Marie Majeure ; ibid., IV, 1976, p. 85 pour Sainte-Sabine.

48. M. Dieulafoy, op. cit. (n. 6), p. 85-86 et fig. 5, p. 85. On notera que sur ce point aussi, Lizop a initialement suivi les interprétations de son maitre ; il a en effet reproduit cette coupe dans sa propre publication, op. cit. (n. 3), fig. 4, p. 36. 


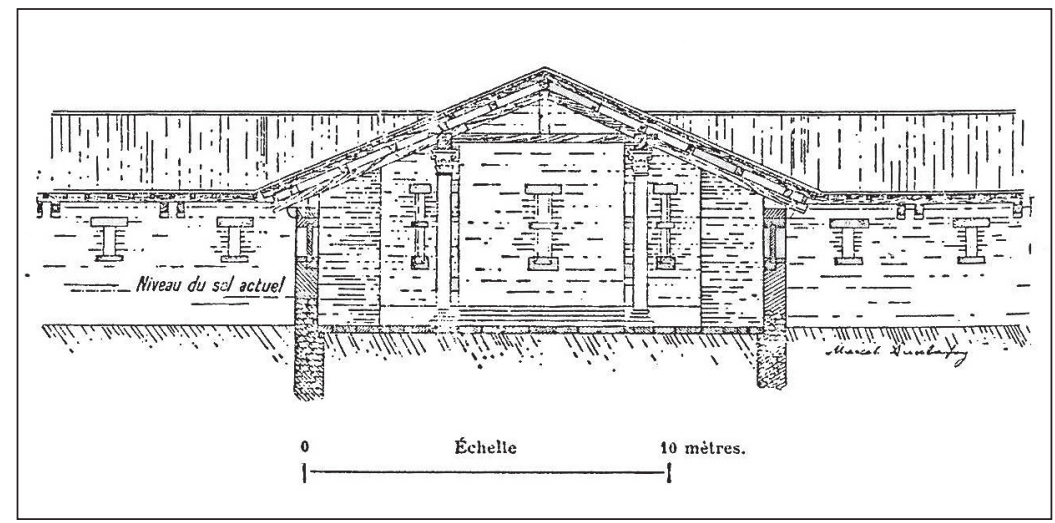

FIG. 10. - Coupe transversale restituée de la basilique chrétienne, d'après CRAI, 1914, fig. 5, p. 85 (dessin M. Dieulafoy).

prolonger la charpente au-dessus de ce portique conduirait en effet à laisser libre une hauteur de 1,75 m seulement au droit de son égout de toiture.

Il faut donc songer à une restitution plus ambitieuse, du type de celle que Bertrand Sapène avait déjà proposée avant cette découverte qui suffit à clore le débat. Il a mis à profit en effet la réédition de son guide du site en 1961 pour l'illustrer d'une vue cavalière de la basilique autrement majestueuse, allant jusqu'à figurer un monument construit en grand appareil ${ }^{49}$ (fig. 11), ce qui n'était évidemment pas le cas. Ce croquis était assez évocateur pour avoir inspiré à une main anonyme une restitution plus élaborée que conserve le musée de Saint-Bertrand (fig. 12). Et si notre propre restitution sous forme d'évocation à l'aquarelle due à Jean-Marie Gassend peut paraître une autre variation sur le même thème (fig. 13), c'est parce que, comme Bertrand Sapène et son épigone, nous avons pris appui pour l'établir sur un corpus de monuments chrétiens bien plus nourri que celui dont disposait Marcel Dieulafoy.

Sur deux points cependant nous nous séparons de nos devanciers. L'un va de soi : l'absence dans cet état primitif de la basilique d'éléments que nous avons jugés adventices : le prétendu « narthex » à l'ouest, le chevet polygonal à l'est, les salles annexes nord. L'autre tient à la couverture du chœur que Bertrand Sapène et Marcel Dieulafoy ont représentée en continuité avec celle de la basilique ; nous 


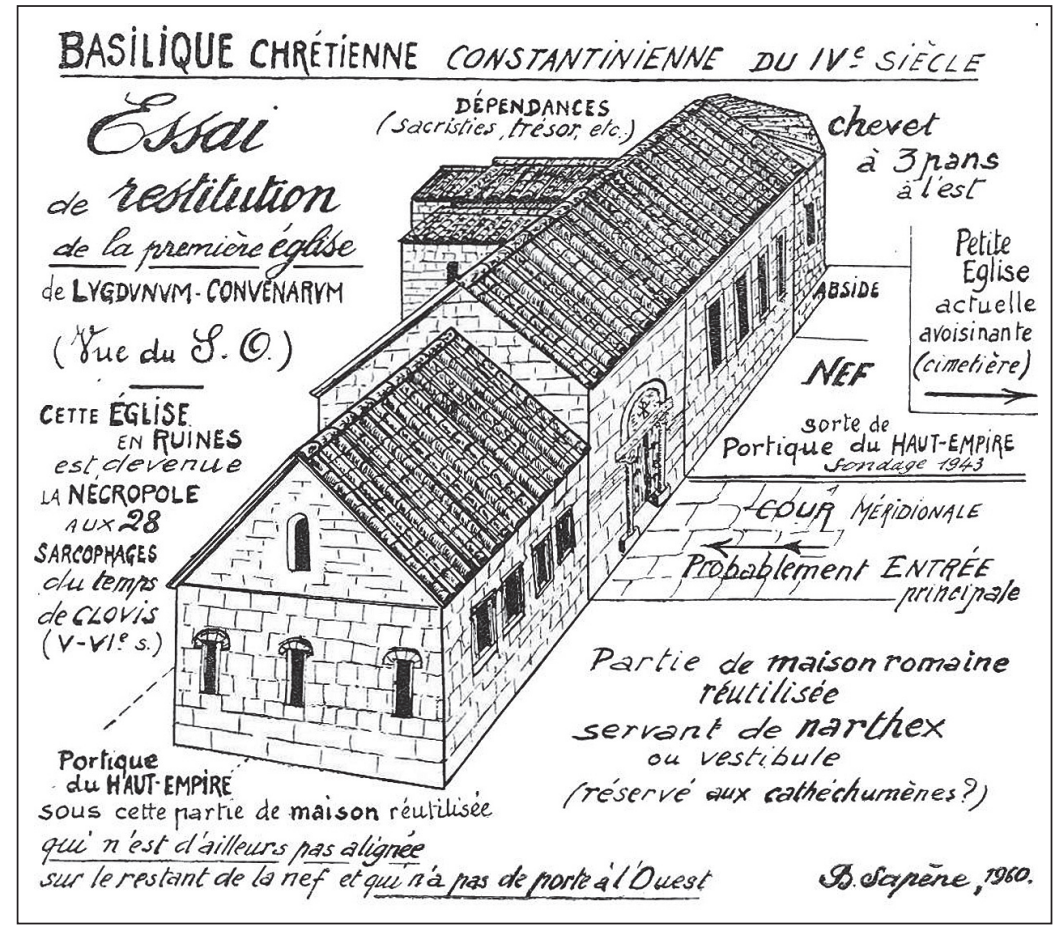

FIG. 11. - Vue cavalière restituée de la basilique chrétienne, d'après B. Sapène, Saint-Bertrand-de-Comminges, Toulouse, 1961, p. 108 (dessin B. Sapène).

avons opté en revanche pour une toiture distincte et plus basse, afin de nous conformer à l'usage le plus général dans le premier art chrétien.

Les autres différences sont minimes. Ainsi à propos de l'élévation de l'édifice pour laquelle nous nous sommes montrés moins audacieux que l'auteur anonyme du dessin du musée en imaginant des murs d'une hauteur d'une douzaine de mètres dans la nef et de $9 \mathrm{~m}$ dans le chœur ${ }^{50}$. Et surtout pour l'éclairage dont on ignore tout dans ce monument arasé au niveau des fondations, de sorte que décider du nombre des baies, de leur forme ou de leurs dimensions est affaire d'appréciation ou de sensibilité personnelles. Il n'est pas

50. Sur le dessin anonyme, l'élévation paraît être en effet de $17 \mathrm{~m}$ environ dans la nef, ce qui n'aurait en soi rien d'invraisemblable : les murs de la nef centrale de Sainte-Sabine, que nous avons déjà invoquée à titre de parallèle, culminent à près de $19 \mathrm{~m}$. Mais la largeur de leurs fondations est de $0,90 \mathrm{~m}$ contre $0,60 \mathrm{~m}$ à Saint-Bertrand, ce qui nous a conduits à adopter à peu de choses près la proposition moins ambitieuse de $\mathrm{B}$. Sapène. 


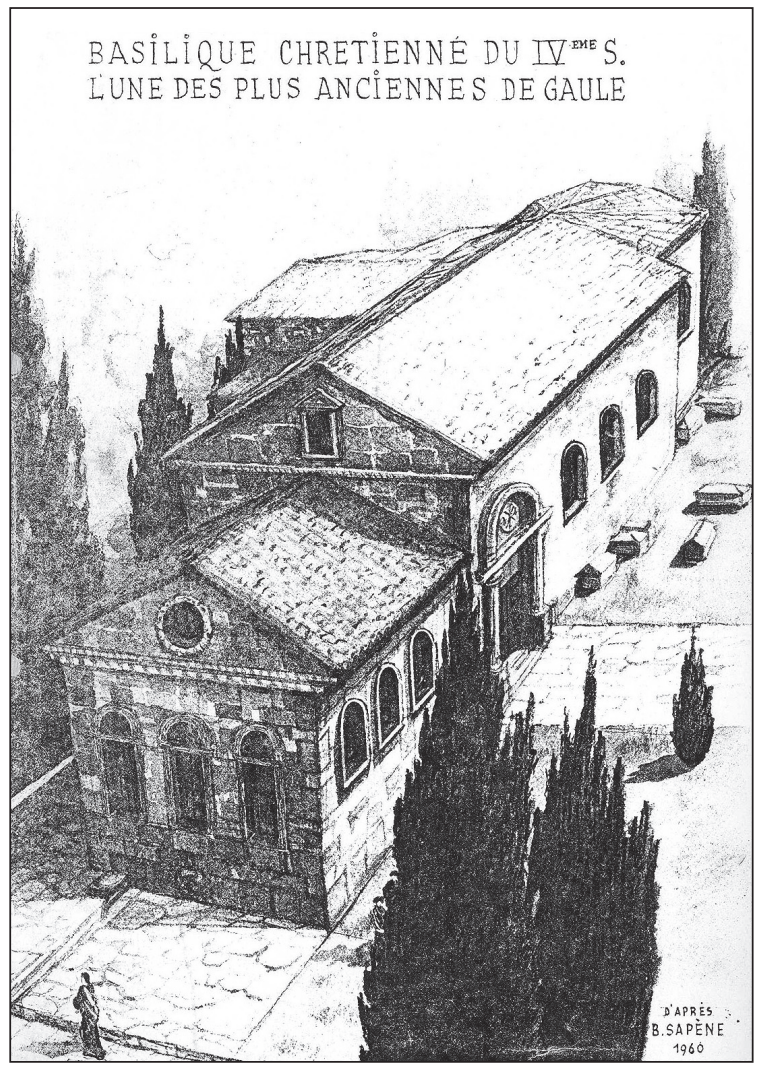

FIG. 12. - Vue cavalière restituée de la basilique chrétienne, dessin anonyme conservé au musée de Saint-Bertrand-de-Comminges.

douteux cependant que cet éclairage était bien plus généreux que ne le supposait Marcel Dieulafoy, pour qui les célébrations tenues dans la basilique auraient baigné dans un clair-obscur empreint d'un certain romantisme, ou plutôt d'un misérabilisme certain ${ }^{51}$. Cela est d'autant plus surprenant qu'il soupçonnait que les baies étaient obturées par des vitraux ${ }^{52}$, chose que paraissent confirmer les

51. M. Dieulafoy, op. cit. (n. 6), p. 90 : « L'éclairage était défectueux, en hiver surtout où le soleil se cache de très bonne heure derrière les hautes montagnes ; mais comme dans la vie journalière la lumière était distribuée d'une manière parcimonieuse, l'assistance se contentait du jour très discret donné par les fenêtres et, la nuit venue, des vagues lueurs répandues par des torches de pins résineux ou quelques brasiers incandescents. »

52. M. Dieulafoy, ibid., p. 89-90: « De petites fenêtres garnies de panneaux de verre à vitres multicolores, montées sur des châssis de bois ou des clôtures de marbre, perçaient les murs. » 


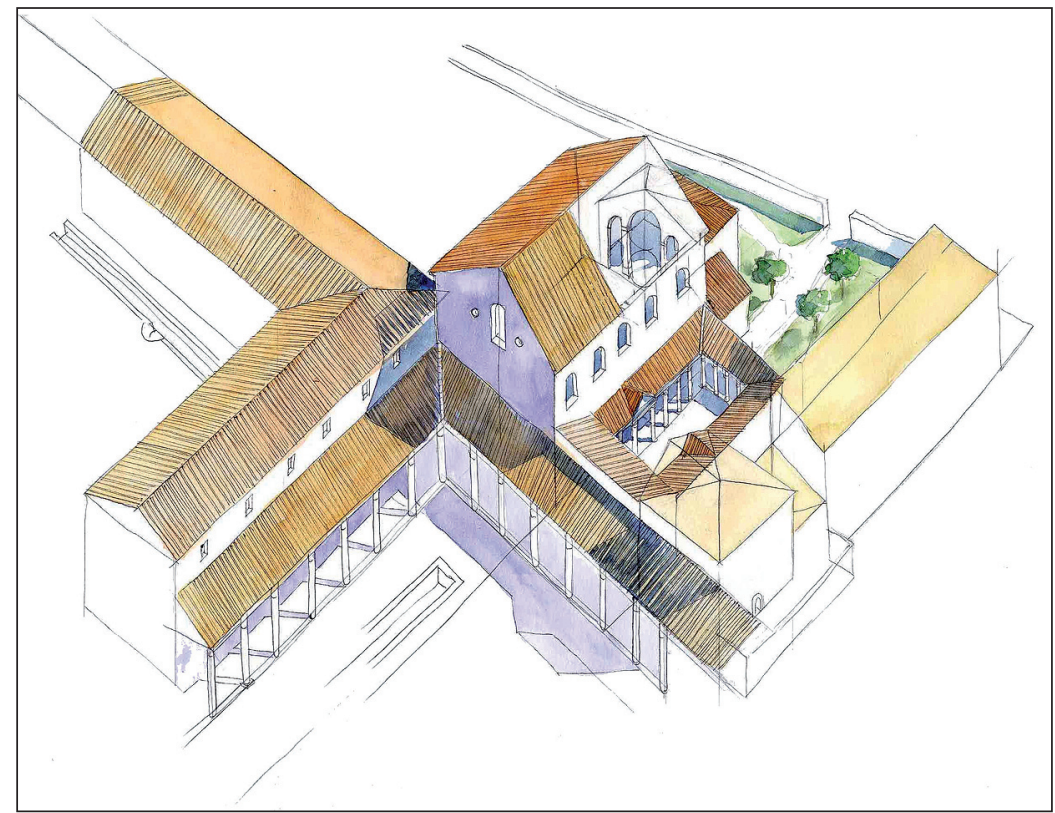

FIG. 13. - Évocation à l'aquarelle du premier état de l'établissement chrétien par Jean-Marie Gassend.

recherches encore en cours de Marie-Thérèse Marty sur le verre issu de nos fouilles.

S'agissant de l'ordonnance intérieure de l'édifice, nos divergences portent essentiellement sur la restitution du chœur. Les premiers fouilleurs le jugeaient être de plain-pied avec la nef, tandis que nous optons pour une surélévation d'un degré dont nous semble garder trace l'altimétrie des sarcophages installés ultérieurement dans la basilique, qui est plus élevée dans le chœur que dans la nef. Et nous croirions volontiers d'autre part que les empreintes des supports de bases conservées à la naissance du chœur (fig. 1, 2, 3 et 9) s'accorderaient mieux à la restitution d'un arc de chœur à trois baies (du type de celui qui figure sur la célèbre représentation de l'ecclesia mater sur une mosaïque de Tabarka ${ }^{53}$ ) qu'à celle d'une barrière de chœur

53. Pour la restitution de ce monument maintes fois étudié, on se bornera à renvoyer à N. Duval, «La représentation du palais dans l'art du Bas Empire et du Haut Moyen Âge d'après le Psautier d'Utrecht », Cahiers archéologiques XV, 1965, p. 244-247, tout en signalant que dans un autre article, "La mosaïque du "Palatium" de S. Apollinaire-le-Neuf représente-t-elle une façade ou un édifice aplani ? ", XXV Corso di Cultura sull'Arte revennate e Bizantina, Ravenne, 5/15 mars 
qui avait les faveurs de Raymond Lizop ${ }^{54}$ (fig. 13). Cela à cause de leur taille apte à porter des fûts de $0,40 \mathrm{~m}$ de diamètre au moins, mais aussi parce que l'existence à la naissance du chœur d'un arc unique dont l'ouverture serait de quelque $9 \mathrm{~m}$ paraît peu compatible avec l'élévation relativement modeste que nous avons retenue pour la basilique.

Du reste, une telle restitution s'accorderait bien avec un édifice dont la décoration était sûrement soignée : un dallage de marbre polychrome sur le sol ${ }^{55}$, de possibles lambris de marbre en partie basse des murs qui étaient sûrement enduits en tout cas $^{56}$. Ce qui fait regretter que rien n'ait été retrouvé des aménagements liturgiques. Tout au plus peut-on suggérer que la table d'autel en usage dans la basilique était sans doute plus ou moins analogue à celle dont deux fragments jointifs ont successivement été retrouvés ces dernières années dans la ville haute de Saint-Bertrand ${ }^{57}$ (fig. 14) car Raymond Lizop signale dans le matériel issu de ses fouilles un éclat de marbre décoré d'un oméga de facture très semblable à celui qui figure sur cette table ${ }^{58}$.

\section{Les annexes méridionales}

La restitution des annexes méridionales de la basilique nous retiendra moins même si elle est grevée d'une incertitude qui tient à l'extension à donner à la cour centrale qui en constitue l'élément majeur. Comme le montrent les tracés en tiretés de la fig. 9, faut-il la prolonger jusqu'à l'épaulement de la nef, voire jusqu'au mur est de l'annexe repérée au sud du chœur, ou lui donner une extension plus

1978, Ravenne, 1978, le même N. Duval, p. 106, laisse ouverte l'interprétation du motif de gauche : « Triple arcade devant l'abside ou devant l'abside et les sacristies?»

54. R. Lizop, op. cit. (n. 4), p. 41.

55. Selon R. Lizop, op. cit. (n. 9), p. 436 ; à noter toutefois que M. Dieulafoy, op. cit. (n. 6), p. 70 et le même R. Lizop, op. cit., p. 36-37 ne signalent que du marbre blanc qui était sans doute le matériau le plus largement utilisé.

56. M. Dieulafoy, op. cit. (n. 6), p. 89 ; R. Lizop, op. cit. (n. 9), p. 436.

57. Pour le premier fragment, consulter R. Gavelle, «Sur une table inédite trouvée à SaintBertrand-de-Comminges et sur les relations de son décor avec la basilique chrétienne du même site ", Revue de Comminges 95, 1982, p. 502 ; R. Favreau, Corpus des inscriptions de la France médiévale, 8, Paris, 1982, n 15, p. 51-52 ; Ch. Deroo, M. Durliat, M. Scelles, Monuments sculptés en France pendant le Haut Moyen Age, IV, Haute-Garonne, Paris, 1987, n ${ }^{\circ} 101$, p. 72-73 ; Pulchra imago - Fragments d'archéologie chrétienne, catalogue d'exposition, Saint-Bertrandde-Comminges, 1991, p. 83-84. Pour l'ensemble du monument, voir désormais la plaquette Une table d'autel pour Convenae rédigée en 2010 par J.-L. Schenck et Y. Narasawa à l'occasion d'une exposition temporaire du musée archéologique départemental de Saint-Bertrand-de-Comminges.

58. R. Lizop, op. cit. (n. 4), p. 49 ; un relevé grandeur nature de ce fragment dû au même R. Lizop est conservé au musée. 


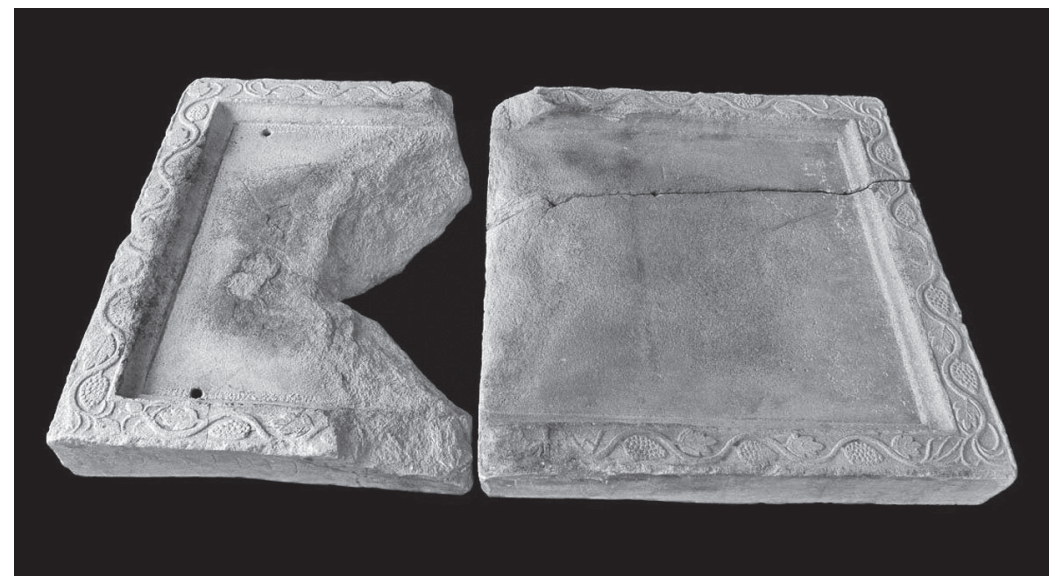

FIG. 14. - La table d'autel conservée au musée de Saint-Bertrand-de-Comminges (cliché K. Schenck-David).

modeste ? Autant de questions à jamais insolubles car la réponse à leur donner ne pourrait venir que d'une fouille à l'intérieur du cimetière du Plan, encore en usage aujourd'hui. Aussi le parti retenu par Jean-Marie Gassend pour son évocation du monument (fig. 13) n'est-il qu'un parmi d'autres possibles.

$\mathrm{Du}$ moins est-il assuré pour la restitution des élévations. Le rythme de la colonnade des portiques nord et sud ne fait aucun doute en effet à cause de la présence dans ses murs stylobates de blocs de marbre en remploi espacés de quelque 1,70 $\mathrm{m}$ d'axe en axe qui lui servaient de support ${ }^{59}$. Quant à sa hauteur, nous l'avons estimée à $3 \mathrm{~m}$ environ, soit une cote analogue à celle de la largeur hors œuvre des portiques. La seule incertitude porte sur le matériau des éléments porteurs de la couverture : bois, pierre ou marbre ? Ces deux dernières solutions ne sont pas à exclure si l'on en juge par le soin porté au traitement des sols des couverts : des tapis de mosaïque polychrome sous le portique méridional et sans doute les pièces de l'aile ouest, dont les tesselles ont été retrouvées en abondance dans nos fouilles ; du marbre peut-être, comme dans la basilique, sous le

59. Sans doute ces supports n'ont-ils été insérés que dans un second temps, afin de mieux asseoir une couverture assise sur de médiocres fondations, mais non sans doute de modifier l'ordonnance de la colonnade, ce qui nous a conduits à retenir pour son état primitif le rythme qu'elle connaissait certainement après cette réfection qui répond à un état I A (fig. 15) dont on dira un mot dans un instant. 
portique nord où toute trace de sol avait disparu. Le sol de la cour, en revanche, n'avait aucun revêtement.

D'une façon ou d'une autre - comment savoir ? -, ces annexes ne pouvaient ouvrir que sur la rue qui longeait par l'est l'îlot au sein duquel est l'établissement chrétien. Elles avaient donc pour objet de commander l'accès à la basilique, ce qui explique le traitement monumental qui leur a été réservé. Mais leur fonction aurait été plus importante encore si elles ouvraient également sur la domus $\mathrm{V}$ comme nous sommes enclins à le croire. En ce cas, elles auraient joué un rôle cardinal de distribution des circulations au sein d'un dispositif où l'essentiel des annexes méridionales de l'édifice de culte serait à rechercher dans cette domus et non dans la cour qui la jouxte (fig. 9). Mais quelle que soit l'extension que l'on choisisse de donner au premier établissement chrétien du Plan, il reste à deviner quelle était sa fonction.

\section{QUELLE FONCTION POUR CET ÉTABLISSEMENT ?}

Pour déterminer la fonction de l'établissement chrétien, il est une donnée à prendre en compte que nous avons passée sous silence jusqu'à présent : la date de sa construction. En la matière, l'éclairage provient non tant du matériel céramique dont la chronologie est assez lâche, mais des très nombreuses monnaies qu'ont livrées les couches du chantier de construction des annexes. Aucune n'est formellement postérieure à la fin $d u \mathrm{IV}^{\mathrm{e}}$ siècle mais la présence en leur sein d'un pourcentage très élevé d'imitations et d'objets monétiformes a conduit Jean-Pierre Bost et Clary Namin à rapporter ces émissions aux années $400-450^{60}$, ce qui invite à placer au plus tôt au tournant du milieu du $\mathrm{V}^{\mathrm{e}}$ siècle la construction de l'établissement chrétien $^{61}$.

Cette date coïncide peu ou prou à Lugdunum Convenarum - ou plutôt à Convenae, puisque tel était désormais le nom de l'agglomération - avec l'achèvement d'un processus majeur de transformation urbaine dont la mise en évidence a été le fait des recherches des dernières décennies. Ce processus a conduit à rien moins qu'une sorte d'inversion de polarité entre les deux éléments constitutifs du

60. J.-P. Bost, Cl. Namin, Les monnaies, Collections du Musée archéologique départemental de Saint-Bertrand-de-Comminges, 5, Saint-Bertrand-de-Comminges, 2002, p. 52-53.

61. Cette date, on le notera, n'est guère différente de celle qui avait été entrevue par J. Hubert, $o p$. cit. (n. 11), qui ne disposait pas cependant de ces données dues aux fouilles récentes. 
chef-lieu des Convènes : une hauteur et son piémont. Cela à cause de la construction sur les hauts de la ville d'un rempart qui a pu être daté par des sondages des environs de 400 et dont les matériaux de construction provenaient - mais moins peut-être qu'il n'a été écrit - des monuments publics du site de plaine dont la plupart paraissent avoir été démantelés sensiblement à même date ${ }^{62}$. Non pourtant que la ville basse ait dès lors été désertée comme suffit à le prouver le long maintien de l'habitat alentour de la basilique et de ses annexes. Mais il est sûr que le pôle le plus dynamique de l'agglomération était désormais le site de hauteur.

Cela suffit à établir ce que n'était sûrement pas l'établissement chrétien : la première cathédrale de la cité. Outre le fait qu'il n'a été retrouvé en son sein ou à ses abords nulle trace d'un baptistère qui était un élément constitutif de tout groupe épiscopal antique ${ }^{63}$, la basilique est de date trop tardive en effet pour avoir été église épiscopale. Après la construction de l'enceinte vers 400, la place d'une telle église n'était pas sur le site de piémont mais sur la hauteur, auprès de nouveaux monuments publics et surtout d'un habitat peuplé de fidèles dont elle avait pour fonction d'assurer la desserte spirituelle. Du reste, c'est bien sur la hauteur que la cathédrale est mentionnée pour la première fois dans le récit du siège de Convenae en 585 que nous a laissé Grégoire de Tours ${ }^{64}$. L'indication peut être tardive, elle n'est pas sans poids car rarissimes sont dans les Gaules les cathédrales dont l'emplacement a varié pendant l'Antiquité65.

En dépit des sarcophages qui ont motivé sa découverte, l'établissement chrétien n'était certainement pas non plus à l'origine

62. Pour une présentation plus détaillée de cette évolution, on renverra aux synthèses des années 1999 et suivantes citées à la n. 20 et pour la construction du rempart et la destruction plus ou moins concomitante des monuments publics de la ville basse aux monographies signalées à la n. 16 .

63. L'argument n'est pas sans réplique car l'établissement n'a pas été fouillé intégralement mais la surface qui a échappé aux investigations paraît trop exiguë pour qu'il ait pu accueillir un baptistère épiscopal dont la surface moyenne dans les Gaules est de l'ordre d'une bonne centaine de mètres carrées : cf. J. Guyon, Les premiers baptistères des Gaules (IVe-VIII siècles), Unione internazionale degli Istituti di Archeologia, Storia e Storia dell'Arte in Rome, Conferenze, 17, Rome, 2000, p. 73.

64. Grégoire de Tours, $H F$, VII, 38, éd. B. Krusch, W. Levison, $M G H, S R M$, I, 1, $2^{\mathrm{e}}$ éd, Hanovre 1951, p. 360. Sur ce récit, voir R. Sablayrolles, «Le siège de Convenae en 585, fiction littéraire et réalité archéologique ou fiction archéologique et réalité littéraire ? ", dans Itinéraires de Saintes à Dougga, Mélanges offerts à Louis Maurin, J.-P. Bost, J.-M. Roddaz, F. Tassaux (éd.), Bordeaux, 2003, p. $151-165$.

65. Les seuls exemples assurés paraissent en effet être ceux d'Arles, Aix-en-Provence et Auxerre : cf. N. Gauthier et J.-Ch. Picard (éd.), Topographie chrétienne des cités de la Gaule des origines au milieu du VIII siècle, II. Provinces ecclésiastiques d'Aix et d'Embrun (Narbonensis Secunda et Alpes Maritimae), Paris, 1986, p. 24-26 ; III. Provinces ecclésiastiques de Vienne et d'Arles (Viennensis et Alpes Graiae et Poeninae), Paris, 1986, p. 80 ; VIII. Province ecclésiastique de Sens (Lugdunensis Senonia), Paris, 1992, p. 53-55. 
un établissement funéraire. Parce qu'elle renvoie à une date plus tardive que le $\mathrm{V}^{\mathrm{e}}$ siècle, la typologie de ces cuves suffirait à le montrer si l'on était assuré que leur chronologie vaut également pour les tombes plus humbles qui les jouxtaient et que l'on est en peine de dater en l'absence du moindre dépôt funéraire. Toute hésitation doit être levée cependant si l'on se rappelle le dense contexte urbain dans lequel l'établissement a été inséré, qui interdit d'imaginer une quelconque promiscuité des vivants et des morts. D'ailleurs, à date haute, c'est à l'écart de l'agglomération, à Saint-Just-de-Valcabrère, qu'il faut imaginer le principal cimetière chrétien, ainsi que l'a bien montré Jean-Luc Schenck ${ }^{66}$.

Ni cathédrale, ni église funéraire, l'établissement chrétien du Plan ne peut être que le siège de la basilique destinée à la desserte spirituelle de la ville basse où elle offre une sorte de contrepoint de la cathédrale implantée sur la hauteur. La situation n'est pas sans rappeler celle d'autres villes auxquelles leur topographie conférait une semblable structure bipolaire. Que l'on songe ainsi à SaintLizier-en-Couserans tout proche dont les concathédrales médiévales, Notre-Dame de la Sède et Saint-Lizier, peuvent avoir succédé à des édifices de culte antiques ${ }^{67}$. Mais autant vaut en Provence pour Riez où la cathédrale installée en ce cas dans la plaine a pour répondant sur la hauteur l'église Saint-Albin que l'évêque Maxime avait construite sur la hauteur dans le second tiers du $\mathrm{V}^{\mathrm{e}}$ siècle $^{68}$. Resterait à savoir qui a eu l'initiative de l'installation sur le piémont d'un autre lieu de culte que la cathédrale : l'évêque ou un évergète chrétien ${ }^{69}$ ? Et que faire de la domus $\mathrm{V}$ qui nous a paru être liée à l'établissement ? La demeure de son desservant ou celle de son fondateur?

66. J.-L. Schenck, « Valcabrère, église Saint-Just », dans Les premiers monuments chrétiens de la France N. Duval (dir.), t. II, Sud-Ouest et Centre, Paris, 1996, p. 201-206.

67. "Saint-Girons - Saint-Lizier-en-Couserans », dans Topographie chrétienne des cités de la Gaule des origines au milieu du vIII siècle, XIII, Province ecclésiastique d'Éauze (Novempopulana), N. Gauthier, B. Beaujard, F. Prévot (éd.), Paris, 2004, p. 83-93 et spécialement p. 92-93.

68. En attendant la parution prochaine des addenda et corrigenda du volume XVI de la collection où seront prises prendra en compte les récentes recherches de $\mathrm{Ph}$. Borgard qui renouvellent fondamentalement l'appréhension de Riez, on reverra à Topographie chrétienne des cités de la Gaule des origines au milieu du VIII siècle, II. Provinces ecclésiastiques d'Aix et d'Embrun (Narbonensis Secunda et Alpes Maritimae), N. Gauthier et J.-Ch. Picard (éd.), Paris, 1986, p. 29-40.

69. Cette dernière hypothèse est à prendre très sérieusement en compte si l'on se rapporte aux travaux des dernières décennies sur l'importance des actes d'évergésie de l'aristocratie convertie : songer pour l'Italie à celui de J.-P. Caillet, L'évergétisme chrétien en Italie et à ses marges, Collection de l'École française de Rome, 175, Rome, 1993 et pour l'ensemble de l'orbis romanus à la synthèse d'Y. Duval et L. Pietri, «Évergétisme et épigraphie dans l'Occident chrétien (IV $-\mathrm{VI}^{\mathrm{e}} \mathrm{s}$.) 》, dans Actes du X $X^{e}$ Congrès international de Nîmes d'épigraphie grecque et latine, Nîmes, 4-9 octobre 1992, Paris, 1997, p. 371-396. 
Autant de nouvelles questions sans réponse dans ce dossier qui n'en manque point.

\section{Les extensions de l'établissement au sixième siècle}

L'établissement chrétien a subi de menues modifications - réfection de la colonnade des annexes méridionales, création de modestes annexes au nord de la basilique (fig. 15) - qui répondent à un état I A sur lequel nous passerons pour faire bref. D'une tout autre importance sont les extensions qu'il a également connues; faute de pouvoir établir précisément leur chronologie relative, nous avons choisi de les rapporter à un état II au sein duquel nous avons en outre distingué deux phases, A et B (fig. 16), qui nous retiendront plus longuement.

\section{La basilique}

Des trois extensions de la basilique, deux n'ont que modérément outrepassé les limites de la parcelle IV. Ainsi au nord où les trois salles accolées au chœur à l'état $\mathrm{I}^{70}$ ont été agrandies à la fois vers le nord et vers l'ouest au détriment de la parcelle III. Pour autant, si leur surface a triplé à l'issue de ces travaux, elle atteint à peine $70 \mathrm{~m}^{2}$ hors œuvre. Vers l'est où l'empiètement a porté sur le domaine public, il est plus modeste encore : une saillie d'un mètre au plus sur la rue riveraine de l'îlot - et ce, sur une longueur de trois à quatre mètres seulement. Le paysage urbain n'a sans doute guère été affecté par cette extension qui a eu pour objet de greffer sur le chevet plat originel un chevet à trois pans aux murs bien appareillés et aux fondations puissantes : profondes d'un bon mètre, elles atteignent jusqu'à 1,40 m de largeur pour le mur est. Aussi est-il un peu surprenant que ce nouveau chevet ait fait place à l'état II B (fig. 16) à un autre chevet de plan analogue, mais de plus modeste envergure puisqu'il est inscrit à l'intérieur du dispositif à trois pans. Faut-il voir là un repentir ? Ou juger que ce rétrécissement visait à éviter les désordres que l'appareil du chevet précédent avait pu connaître parce qu'il chevauchait le mur de clôture dérasé ? On ne sait.

Laissons cela pour en venir à la prolongation de la basilique vers l'ouest qui est autrement importante : elle couvre une surface de 


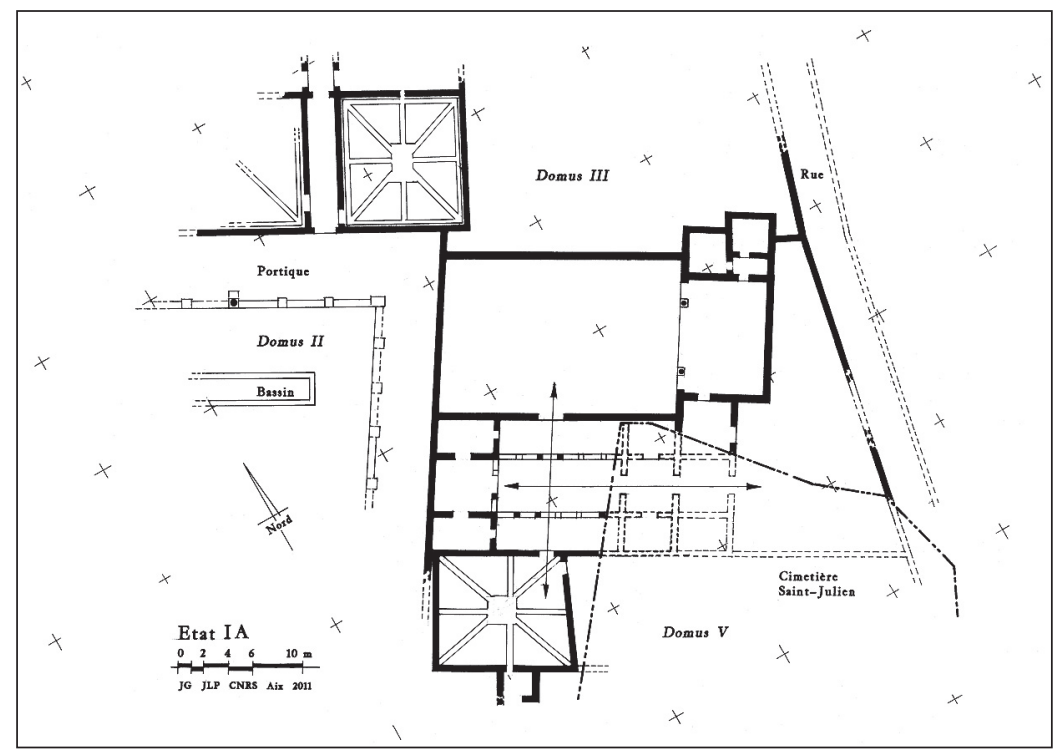

FIG. 15. - L'état I A de l'établissement chrétien (dessin J.-L. Paillet).

près de $150 \mathrm{~m}^{2}$ à laquelle il convient d'ajouter une surface sensiblement égale pour l'extension concomitante des annexes méridionales comme on le dira bientôt. Cet espace gagné sur la domus II reste cependant modeste si on le rapporte à l'aire considérable couverte par cette domus dont il n'occulte en outre qu'une minime part du seul jardin. Le corps d'habitation n'a en rien été affecté ; bien plus, il a été veillé à en conserver l'accessibilité ${ }^{71}$ et à n'aménager aucune communication avec l'édifice de culte agrandi ${ }^{72}$. Autant d'éléments qui montrent que la domus était encore habitée, chose que nos fouilles ont d'ailleurs confirmée. Mais c'est aussi la preuve qu'à la différence de ce que nous supposons pour la domus $\mathrm{V}$, la demeure est restée jusqu'à sa fin indépendante de l'établissement chrétien.

71. Comme le montre le fait que le mur nord de l'extension de la basilique n'est pas dans l'exact prolongement du mur gouttereau de la nef, mais parallèle à la façade méridionale du corps d'habitation de la domus. Cela afin de ménager une sorte de corridor de $3 \mathrm{~m}$ environ de largeur à hauteur de son accès.

72. Sans doute R. Lizop avait-il noté que « le mur de façade [i. e. : le mur ouest] (...) semble avoir été percé d'une porte ; peut-être faut-il rapporter à celle-ci une longue dalle de marbre percée de trous (pour deux montants ?) découverte au voisinage en 1920 » (P. Lavedan, R. Lizop, B. Sapène, op. cit [n. 2], p. 103) mais l'examen des élévations encore largement conservées dément ce jugement. La chose n'avait d'ailleurs pas échappé à B. Sapène qui n'a restitué aucune ouverture à l'ouest sur sa vue cavalière restituée de la basilique (fig. 11). 


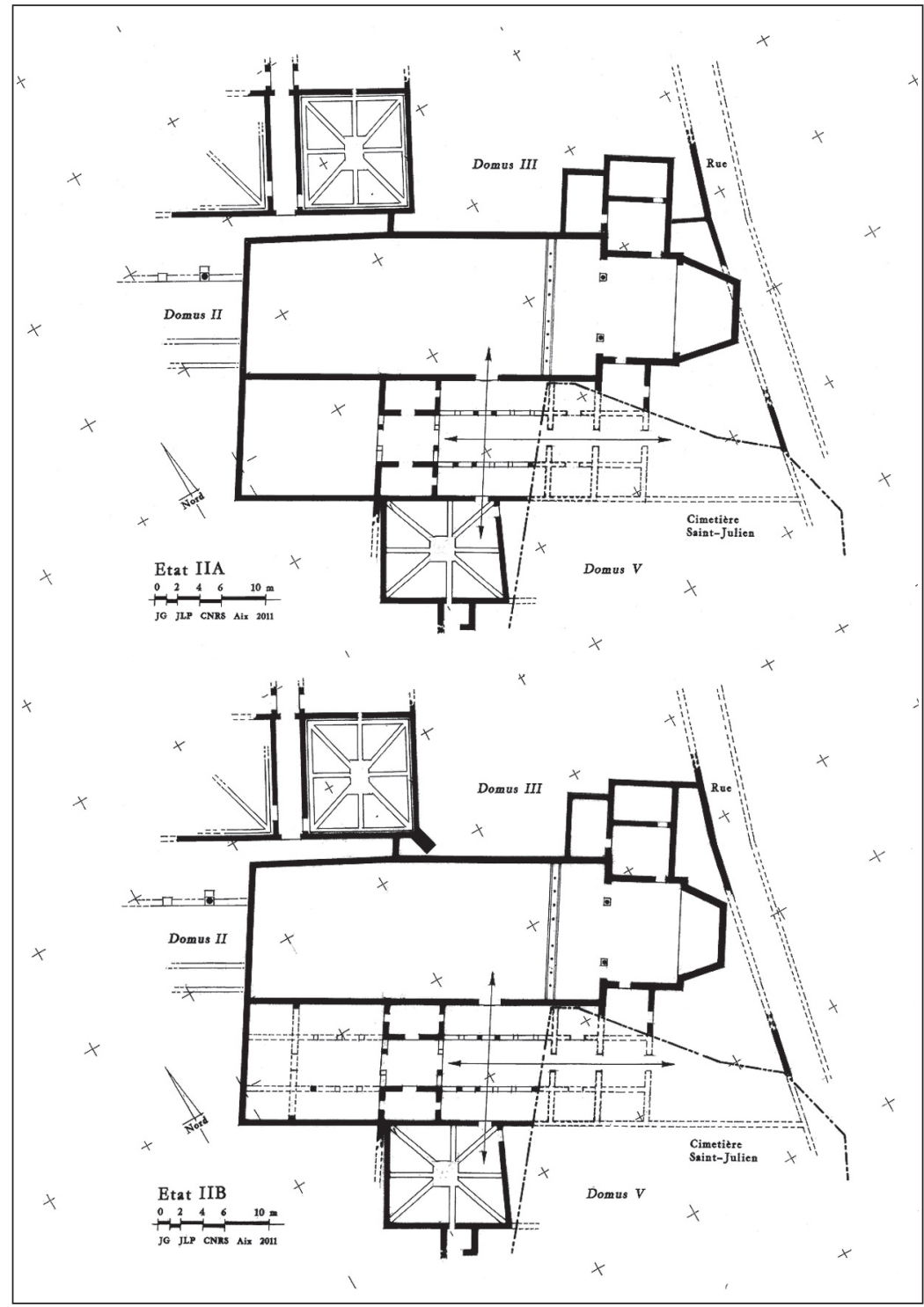

FIG. 16. - Les états II A et II B de l'établissement chrétien (dessin J.-L. Paillet).

Reste à établir la raison d'être de l'extension occidentale de la basilique qui affecte la forme d'un carré légèrement déformé de quelque 12 m de côté dans œuvre. Raymond Lizop, on s'en souvient, voyait dans ce dispositif « une pièce bien distincte (qui) ne peut être que le 
narthex ${ }^{73}$. Deux raisons interdisent cette interprétation. L'une est que l'appareil du mur nord de l'extension est chaîné en élévation avec celui de la basilique à hauteur du mur oriental de ce prétendu narthex (l'ancien mur mitoyen des parcelles II et IV) qui avait donc préalablement été détruit en élévation, ce que confirme d'ailleurs le fait qu'il a été retrouvé arasé au niveau des fondations de la basilique lors de sa fouille. Il faut dès lors tenir l'extension comme une prolongation de la nef primitive, donc lui attribuer contrairement à nos devanciers (fig. 11 et 12) même élévation et même couverture qu'elle (fig. 17).

L'autre raison est que, si la nef a été étendue vers l'ouest, elle a probablement été amputée à l'est dans le même temps. Comment interpréter en effet le mur qui barre transversalement son vaisseau à $5 \mathrm{~m}$ environ de distance de son extrémité orientale, sinon comme la fondation d'une barrière de chœur? Que cette barrière réponde à une adjonction ne fait aucun doute ${ }^{74}$, non plus que sa raison d'être. Son installation a conduit à ne pas étirer exagérément la nef dont la surface a cependant été accrue d'un bon tiers pour atteindre $315 \mathrm{~m}^{2}$ environ. Mais elle a également contribué à accroître la superficie du chœur d'une soixantaine de mètres carrés auxquels il faudrait ajouter, si les deux modifications ont bien été simultanées, les quelque $34 \mathrm{~m}^{2}$ dus à la greffe d'un chevet à pans coupés sur le chevet plat originel. L'ordonnance générale de l'édifice n'a guère été affectée par ces transformations. Mais il en va autrement de l'équilibre entre ses divers éléments. La proportion entre la nef ouverte aux fidèles et le chœur dont l'accès était réservé au clergé est passée en effet de quelque 4,25 à 1 dans l'édifice de culte primitif à 2,12 à la phase II de son histoire.

\section{Les annexes méridionales}

Les annexes méridionales ont connu vers l'ouest une extension comparable à celle de la basilique. Il a suffi pour y pourvoir de prolonger d'une douzaine de mètres vers l'ouest leur mur méridional et de le lier à un mur perpendiculaire faisant retour vers la façade occidentale de l'édifice de culte contre laquelle il prend appui ${ }^{75}$.

73. R. Lizop, op. cit. (n. 7), p. 244.

74. Ses fondations prennent en effet appui contre les murs gouttereaux de l'édifice primitif dont tous les éléments sont en revanche chaînés.

75. Ce collage est à interpréter comme une pratique de chantier, non comme la marque d'une postériorité. Il se vérifie également pour l'état primitif des annexes, certainement contemporain de la création de la basilique. 


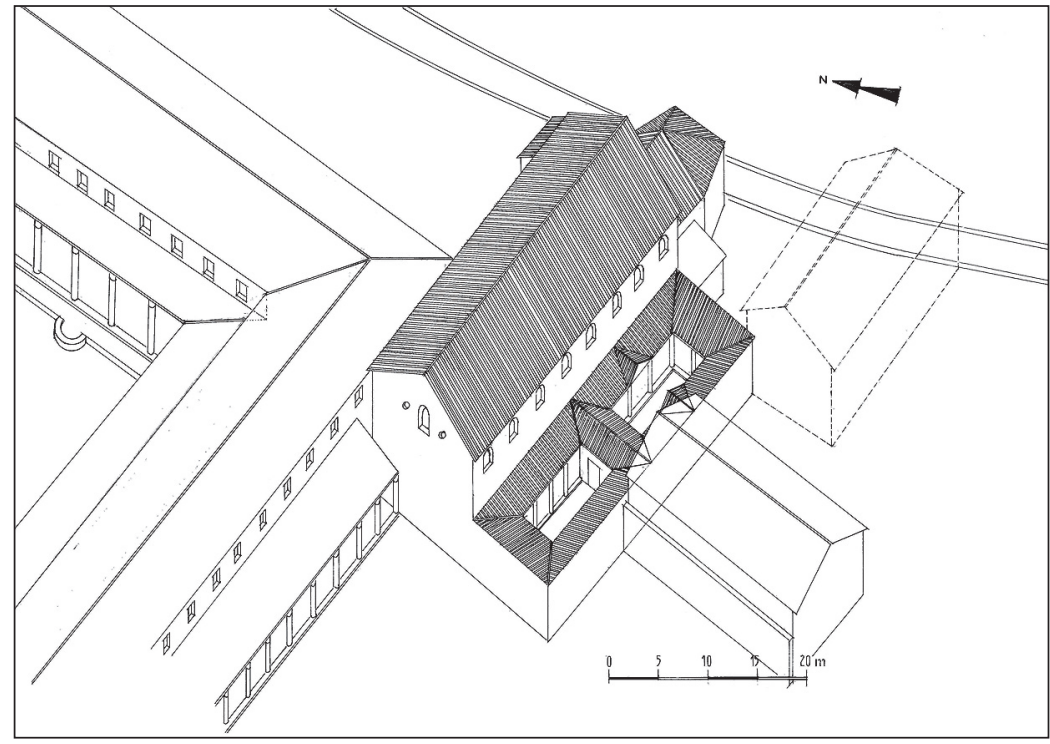

FIG. 17. - Axonométrie du deuxième état de l'établissement chrétien (dessin M.-C. Monguillan).

Ainsi a été constituée une aire de quelque $150 \mathrm{~m}^{2}$ de superficie qui était sans doute originellement une cour ou un jardin accessibles depuis les annexes car, comme la basilique, elle ne paraît pas avoir été en communication avec la domus II au détriment de laquelle elle a été établie ${ }^{76}$ (fig. 16, II A).

Dans un second temps, II B, des murs longitudinaux ont été élevés dans le prolongement des murs stylobates des annexes primitives. Leurs fondations sont plus médiocres encore que celles de leurs devanciers puisque leur hauteur n'excède pas une vingtaine de centimètres, mais leur fonction était identique. La preuve en est donnée par la présence sur l'un d'entre eux d'une empreinte de mortier destinée au scellement d'un support disparu, qui est analogue à celles que nous avons identifiées plus à l'est. Analogue, mais non identique car il s'agit d'un rectangle de $0,42 \mathrm{~m}$ x $0,36 \mathrm{~m}$ et non d'un carré de $0,40 \mathrm{~m}$ de côté. L'axe de cette empreinte, d'autre part, est à $6,38 \mathrm{~m}$ de distance de celui du mur occidental des annexes primitives, ce qui indique que le rythme de la colonnade qu'elle

76. Le doute reste permis toutefois car les sondages sur le mur ouest des annexes n'ont livré que ses fondations. 
supportait était différent de celui des portiques de l'établissement primitif où l'entraxe des supports est de 1,70 m environ : 6,38 n'est pas un multiple de 1,70 (fig. 16, II B).

Ces données invitent à restituer au sud de la basilique non une cour longiligne, mais deux cours séparées par le corps de bâtiment occidental des annexes primitives, certainement transformé pour l'occasion. Que ces cours aient eu même largeur - 4, 25 m environ - est hors de doute mais leur longueur est plus problématique. Nous l'avons déjà dit pour la cour orientale créée dès l'état I de l'établissement ${ }^{77}$; pour la cour occidentale, doit-on penser qu'elle occupait tout le secteur médian de l'extension, profond d'une douzaine de mètres ? Ou, comme nous le croirions volontiers, faut-il imaginer qu'à l'instar de sa voisine elle était bordée à l'ouest par un corps de bâtiment ${ }^{78}$, ce qui réduirait sa longueur à 7,60 m (fig. 16, II B et 17) ? Nul doute en tout cas que dans les annexes comme dans la basilique, l'extension a conduit à une monumentalisation accrue de l'établissement chrétien.

\section{Une évolution caractéristique du sixième siècle}

Les données de fouille sont trop lâches pour dater la deuxième phase de l'établissement, mais le recours peut venir de la nature des transformations qui ont alors affecté la basilique. Elles ont eu pour principal effet, on l'a vu, de donner au chœur une importance sans commune mesure avec celle de l'état I. En cela, elles participent d'un phénomène très répandu dans le premier art chrétien sur lequel les recherches récentes, attentives à repérer les plus minimes modifications, ont opportunément attiré l'attention. Les fouilles exemplaires de Charles Bonnet dans le groupe épiscopal de Genève en offrent sans doute le meilleur exemple ${ }^{79}$. Dans chacun des nombreux édifices de culte que compte ce groupe, le chœur a toujours connu au fil du temps des projections au sein de la nef, d'une importance sans cesse accrue. Pour la seule période antique ${ }^{80}$, c'est au $\mathrm{VI}^{\mathrm{e}}$ siècle

78. Cette hypothèse tient à la présence de deux pilastres adossés contre les murs latéraux de l'extension des annexes à même distance $-4 \mathrm{~m}$ environ - de leur façade occidentale. Que ces pilastres aient eu pour objet de marquer une césure au sein des portiques latéraux de la cour n'est pas douteux ; que cette césure réponde à la façade d'un corps de bâtiment est plus problématique.

79. Dans 1'attente d'une publication définitive d'ailleurs imminente, on consultera Ch. Bonnet, « Les fouilles de l'ancien groupe épiscopal de Genève (1976-1993) », Cahiers d'archéologie genevoise 1, Genève, 1993.

80. Car les époques ultérieures ont connu d'autres transformations hors de notre propos. 
que se situe l'acmé de cette évolution qui répondait sans doute à des nécessités liturgiques (fig. 18). L'hypothèse est d'autant plus vraisemblable que les bâtiments construits ou radicalement réaménagés ailleurs au cours de ce siècle présentent ordinairement eux aussi des chœurs qui empiètent sur la nef. Et ce, qu'il s'agisse d'édifices hors du commun comme celui que Marc Heijmans fouille présentement à Arles ${ }^{81}$ ou de bâtiments plus modestes comme la chapelle funéraire que Pantagathus avait construite à Vienne vers $540^{82}$.

Sans que l'on puisse se montrer plus précis, cela invite à rapporter au $\mathrm{VI}^{\mathrm{e}}$ siècle les extensions de l'établissement chrétien. D'autant qu'elles témoignent d'un autre trait caractéristique de ce siècle : une emprise accrue de l'Église sur le patrimoine foncier, voire sur le domaine public, que le rapprochement des fig. 13 et 17 suffit à mesurer. Initialement cantonné à la parcelle IV, l'établissement a connu une croissance tous azimuts et son édifice de culte, relativement modeste à l'origine, a acquis de ce fait une ampleur digne d'une cathédrale $^{83}$. Mais sa fonction n'a sans doute pas varié : assurer la desserte spirituelle de la ville basse encore habitée. Ce qui n'exclut pas que des tombes aient pu prendre place dans le courant du siècle sous son couvert ou celui de ses annexes. Mais le phénomène n'est bien perceptible qu'au cours des siècles suivants où il a pris une telle ampleur qu'il a conduit à une véritable mue de l'établissement.

\section{La mue de l'établissement en établissement funéraire à partir du septième siècle}

En première analyse, le dernier état de l'évolution de l'établissement peut passer pour une version épurée de son état II. Plus de césure dans la basilique où la barrière de chœur a disparu, mais non peut-être le degré qui la supportait ${ }^{84}$. Et ses annexes méridionales

81. M. Heijmans, «Les fouilles de l'église paléochrétienne de l'enclos Saint-Césaire à Arles », Comptes rendus des séances de l'Académie des Inscriptions et Belles-Lettres, 2008, p. 1191-1205.

82. Si l'on retient l'identification très convaincante de ce monument avec l'église SaintGeorges qui a été proposée par M. Jannet-Vallat, "Vienne, basilique Saint-Pierre, église SaintGeorges ", dans Les premiers monuments chrétiens de la France, t. I, Sud-Est et Corse, N. Duval (éd.), Paris, 1995, p. 254-266 et spécialement p. 263-264 pour Saint-Georges.

83. Sa longueur de $45 \mathrm{~m}$ est en effet voisine de celle de la plupart des cathédrales antiques des Gaules (cf. N. Duval, « L'architecture cultuelle» dans Naissance des arts chrétiens, Atlas des monuments paléochrétiens de la France, Paris, 1991, p. 192) et supérieure à celle de la partie romane de l'actuelle cathédrale de Saint-Bertrand-de-Comminges.

84. Les fondations de ce degré ont en effet été retrouvées par nos prédécesseurs très largement détruites par l'installation sous le sol de la basilique d'un sarcophage et (probablement) d'autres 


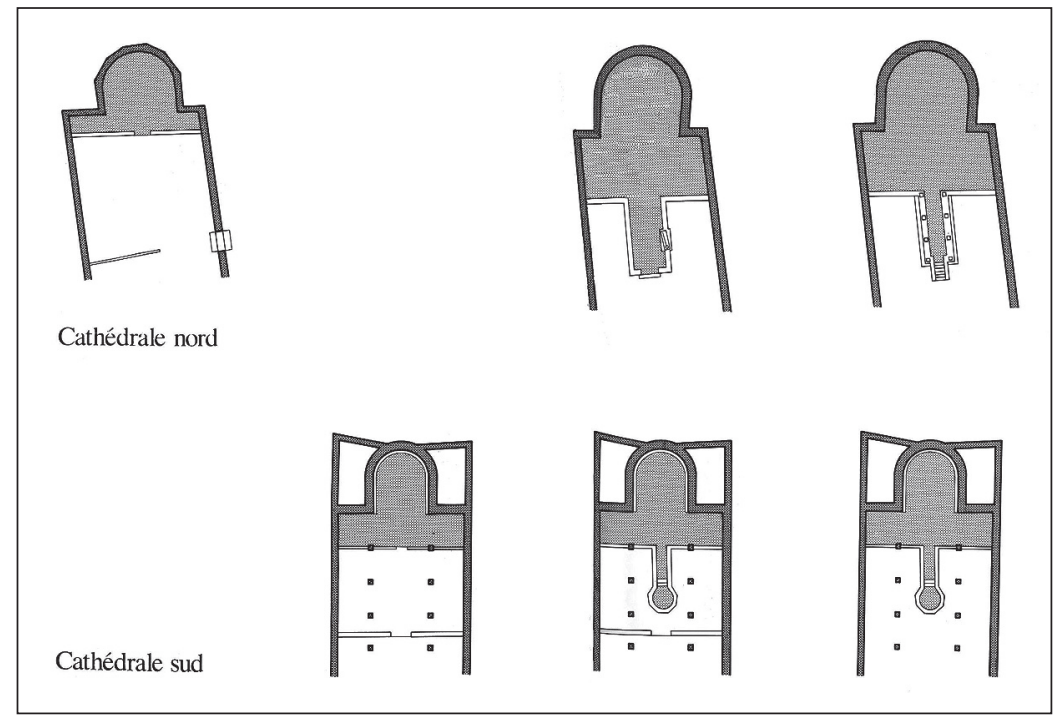

FIG. 18. - L'évolution des installations liturgiques du groupe épiscopal de Genève d'après Ch. Bonnet : la cathédrale nord date de la fin du IV e siècle, la cathédrale sud du courant du $\mathrm{V}^{\mathrm{e}}$ siècle ; les états ultérieurs répondent pour les deux bâtiments aux $\mathrm{V}^{\mathrm{e}}-\mathrm{VI}^{\mathrm{e}}$ siècle et $\mathrm{VI}^{\mathrm{e}}-\mathrm{VII}{ }^{\mathrm{e}}$ siècle.

comptent un unique portique et une unique cour en lieu et place du dispositif très élaboré de l'état précédent (fig. 19). Pour autant, ce dépouillement affiché tient moins à des préoccupations esthétiques qu'à des nécessités fonctionnelles. Il trahit la volonté de transformer un établissement jusqu'alors dévolu à la cura animarum des fidèles vivants en un « campo santo » destiné à recevoir les restes de leurs proches défunts.

Emprunter cet italianisme à des édifices illustres du Moyen Âge toscan est pour nous une façon de marquer que cet ultime état des édifices du Plan est non moins monumental et non moins soigné que les précédents. Cela vaut naturellement pour la basilique qui n'a connu que la menue modification que nous avons dite, mais plus encore pour le portique qui la longe au sud. Sa largeur de $4 \mathrm{~m}$ hors œuvre est sensiblement supérieure à celles des portiques des états précédents et ses fondations sont également plus profondes, de l'ordre de $0,40 \mathrm{~m}$. Il présente en outre à hauteur de l'accès à la 


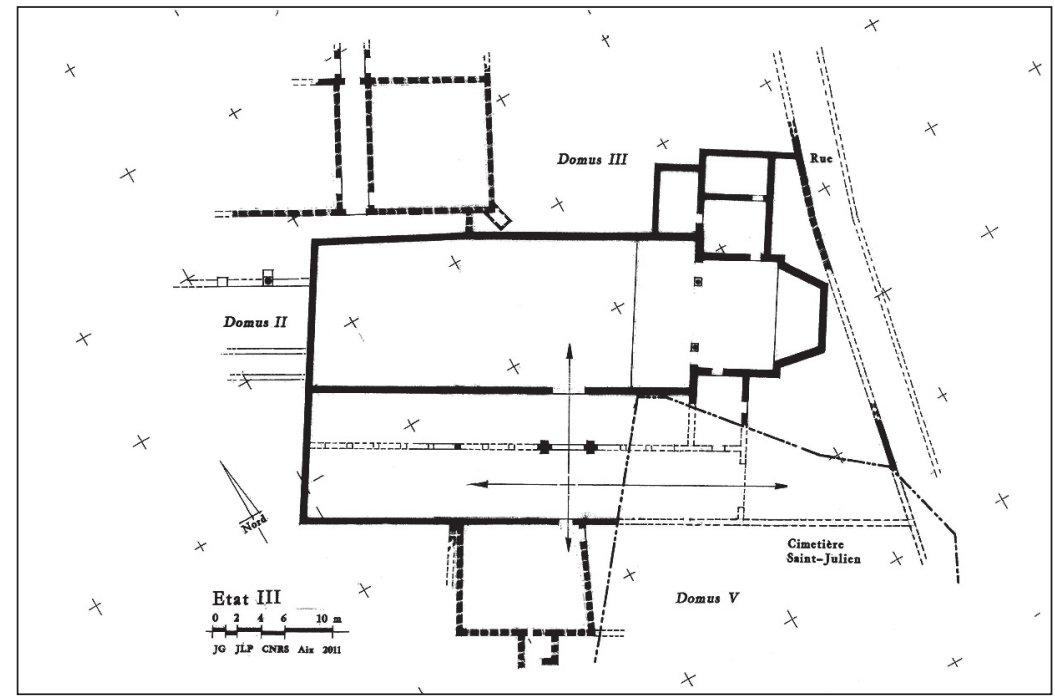

FIG. 19. - L'état III de l'établissement chrétien (dessin J.-L. Paillet).

basilique un seuil large de 2,75 m encadré par de puissants piliers cruciformes. Tous ces éléments imposent d'imaginer pour lui une élévation plus importante que celle de ses devanciers (fig. 20). Aussi n'est-il pas étonnant que les six sarcophages identifiés dans les annexes aient tous trouvé place sous son couvert. Leur nombre reste pourtant minime si on le rapporte aux vingt-sept cuves aujourd'hui conservées dans la basilique, qui ne constituent certainement qu'une partie des sarcophages qu'elle avait accueillis dans 1'Antiquité ${ }^{85}$. Ainsi se dessine au sein du " campo santo » une hiérarchisation des espaces, depuis le moins recherché - la cour - jusqu'au plus âprement disputé : la basilique.

Pour dater ce dernier état de l'établissement chrétien, on ne dispose que d'un indice à la vérité assez problématique, mais il faut bien faire avec ce que l'on a. Il tient à un sarcophage relevant du portique que deux arguments nous paraissent désigner comme l'une des premières tombes à avoir été installée sous son couvert : sa localisation dans un emplacement éminemment privilégié, auprès $\mathrm{du}$ pilier cruciforme occidental du seuil et au long du stylobate de la colonnade, mais aussi le fait que le comblement de la fosse

85. Comme en témoigne un élément de couvercle dépareillé également conservé dans cet édifice qui a certainement été intensément spolié - et ce à toute époque. 


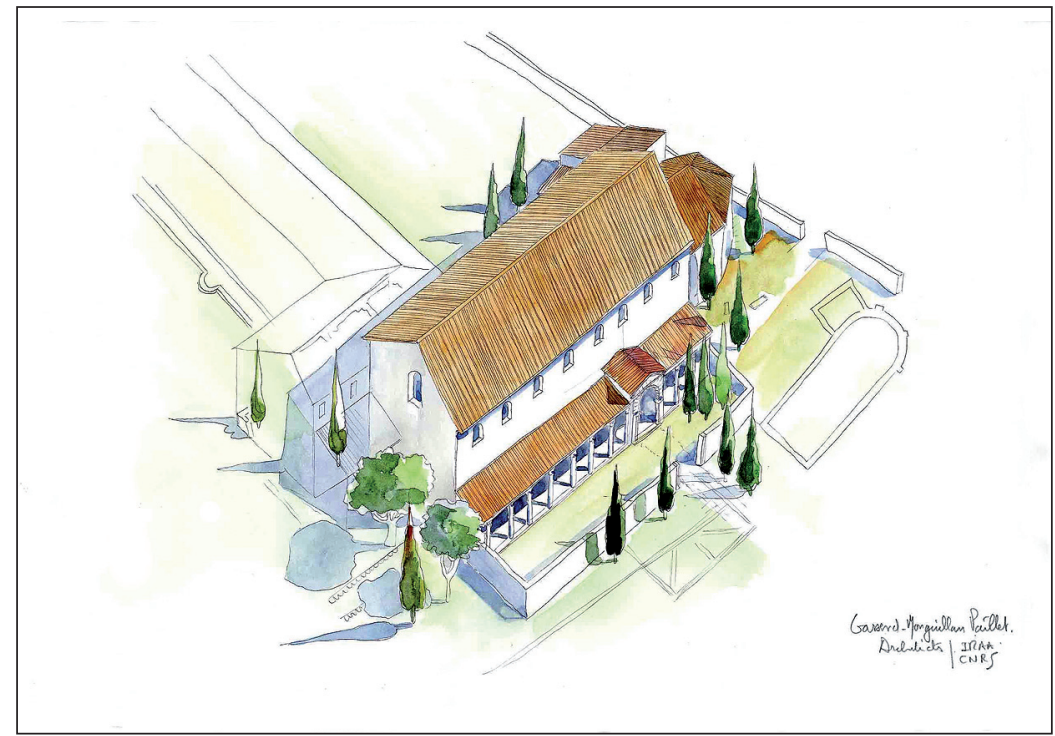

FIG. 20. - Évocation à l'aquarelle du troisième état de l'établissement chrétien par Jean-Marie Gassend.

creusée pour son installation compte des éléments d'architecture en marbre analogues à ceux qui ont été remployés en abondance dans les fondations du portique. Aussi avons-nous fait procéder à la datation par la méthode du radiocarbone des restes osseux qu'il contenait. Elle conduit à placer leur déposition au sein d'un intervalle qui couvre les années 661-873, avec des maxima de probabilité alentour de 690 et $750^{86}$. Ce qui invite à dater dans la première moitié du $\mathrm{VIII}^{\mathrm{e}}$ siècle, date ronde, les transformations qui ont consacré la mue de l'établissement en établissement funéraire.

Consacré, mais non inauguré car cette mue avait commencé dès le $\mathrm{VII}^{\mathrm{e}}$ siècle, voire la fin du $\mathrm{VI}^{\mathrm{e}}$ siècle comme le suggère une autre datation de sépulture par la méthode du radiocarbone ${ }^{87}$. L'étude de l'altimétrie des quelque cent trente tombes qui ont été fouillées au sein des annexes, dans laquelle nous n'avons pas le loisir d'entrer ici, ne permet guère d'en douter car elle met en évidence leur

86. Échantillon Ly-6446 analysé par le Centre de datation par le radiocarbone de l'Université Claude Bernard-Lyon I.

87. Il s'agit de l'échantillon Ly-6445 relatif à une tombe rattachée à l'état II de l'établissement dont la datation est à placer dans l'intervalle des années 457 et 637 , avec des maxima de probabilité en 594 et 570 . 
étagement sur trois niveaux à rattacher à des exhaussements du sol qui répondent respectivement aux états II et III de l'établissement et à son abandon.

L'investissement par des tombes paraît cependant avoir été modeste à l'état II et n'avoir touché que les abords de la basilique, soit le portique nord de ses annexes méridionales. Tout se passe comme si l'on avait voulu conserver la plus longtemps possible à l'établissement sa fonction première. Mais dès lors qu'acte eut été pris qu'il avait mué en établissement funéraire et que l'on eut procédé en conséquence aux transformations de l'état III, les sépultures ont couvert tout l'espace disponible, cour et portiques à la fois, sans compter, naturellement, la basilique. Bien plus, les inhumations n'ont pas cessé, loin de là, lorsque les annexes sont tombées en ruine sans doute au tournant du $\mathrm{X}^{\mathrm{e}}$ siècle si l'on se fie à nouveau à une datation par la méthode du radiocarbone ${ }^{88}$. Et même si ces tombes tardives sont installées dans une couche qui contient des gravats en abondance, elles conservent une disposition ordonnée, presque celle d'un « cimetière en rangée ». En quoi elles préfigurent l'actuel cimetière du Plan, tant il est vrai que la fonction funéraire n'a jamais cessé sur ce site depuis quatorze siècles.

La similitude entre Antiquité et époque contemporaine ne s'arrête d'ailleurs pas là, car de même que la basilique était au cœur du premier établissement chrétien, il demeure aujourd'hui au cœur du cimetière une chapelle romane, Saint-Julien. Qu'il y ait eu un « passage de relais » entre ces deux édifices n'est pas douteux ; l'incertitude ne porte que sur le fait de savoir s'il s'est fait ou non sans hiatus car nos prédécesseurs n'ont laissé aucune indication sur la date de la ruine de la basilique qui peut avoir été conservée-mais pendant combien de temps ? - après la disparition de ses annexes. Il n'importe : que ce soit lato ou stricto sensu, la «blanche robe » des églises romanes qu'il est donné de voir à Saint-Bertrand-deComminges sur le site du Plan comme à Saint-Just de Valcabrère ou dans la cathédrale sur la hauteur n'est que le ravaudage de celle qu'avait déjà revêtue Convenae au cinquième siècle quand l'Église eut choisi de la doter d'une parure monumentale chrétienne.

88. Celle de l'échantillon Ly-2249 relatif à une des tombes les plus superficielles dont la datation est à placer dans l'intervalle des années 790-987, avec des maxima de probabilité en 893, 940 et 830 . 